\title{
FLUORESCENCE STUDIES AND SENSING APPLICATION OF CARBON NANODOTS
}

\author{
A Thesis \\ Presented to \\ the Faculty of the Graduate School \\ at the University of Missouri-Columbia \\ In Partial Fulfillment \\ of the Requirements for the Degree \\ Master of Science \\ by \\ Yuchen Wang \\ Dr. Sheila Baker, Thesis Supervisor \\ December 2013
}


The undersigned, appointed by the dean of the Graduate School, have examined the thesis entitled

\section{FLUORESCENCE STUDIES AND SENSING APPLICATIONS OF CARBON NANODOTS}

presented by Yuchen Wang,

a candidate for the degree of Master of Science,

and hereby certify that, in their opinion, it is worthy of acceptance.

Professor Sheila N. Baker

Professor Paul C.H. Chan

Professor Gary Baker 


\section{DEDICATION}

I would like to dedicate this to my dear parents, families and friends that helped and encouraged me throughout the years. I could not have this done without you. 


\section{ACKNOWLEDGEMENTS}

I would like to express my deepest gratitude to my advisor, Dr. Sheila N. Baker for her guidance, encouragement, support and patience throughout the past two years and half. Her guidance has been of great importance to me in my academic and daily life.

I am also thankful to Dr. Paul C.H and Dr. Gary Baker for their willingness to be my thesis committee members and for their various forms of support during my graduate study. I am also grateful to all the members of Dr. Sheila N. Baker's research group especially Carrie, who has been there to help me from the very beginning. 


\section{TABLE OF CONTENTS}

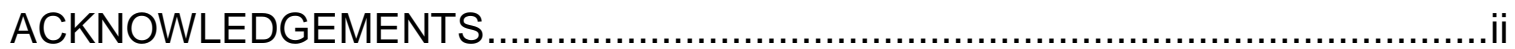

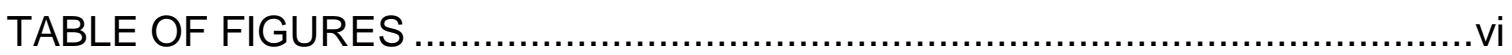

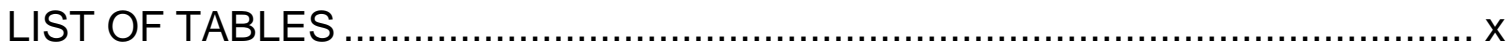

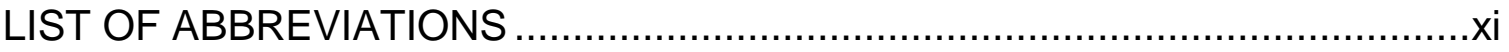

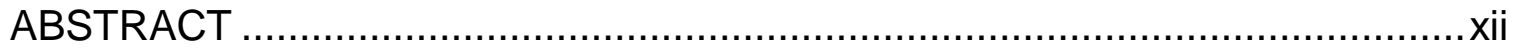

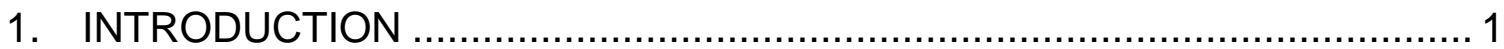

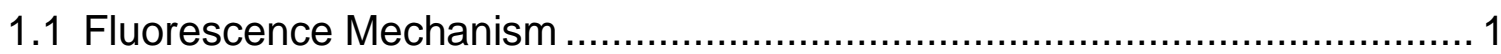

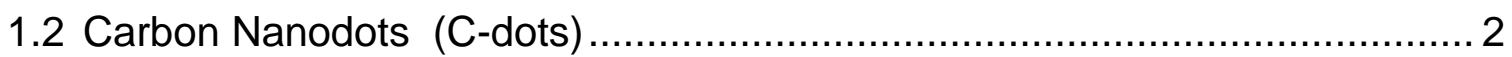

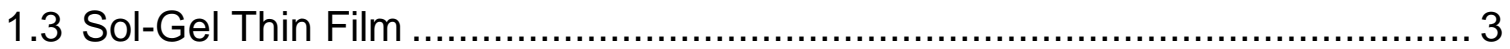

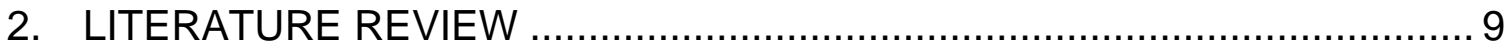

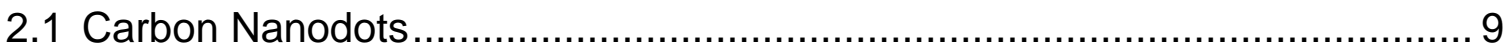

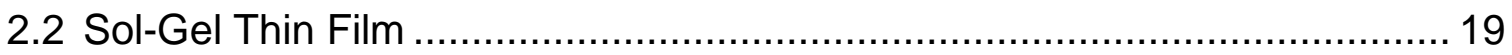

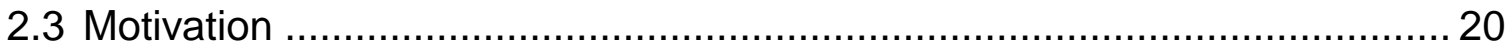

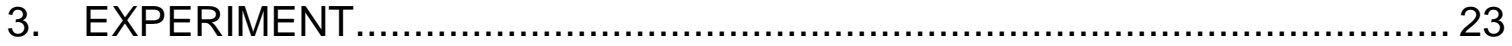

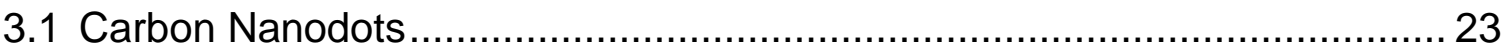

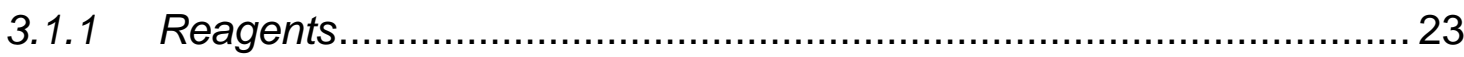

3.1.2 Synthesis of JEFFEMINE® T3000 and T5000 Carbon Nanodots (C-

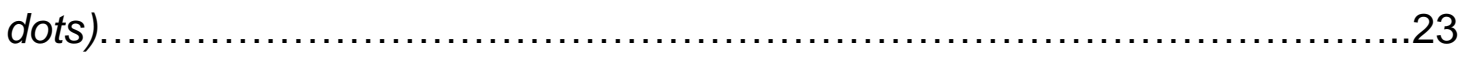


3.2 Carbon Nanodots immobilized in Sol-Gel Thin Films 25

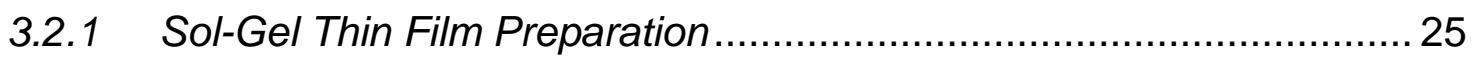

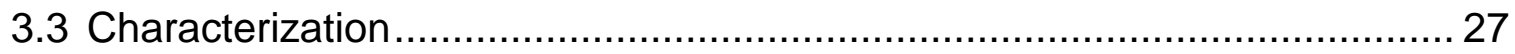

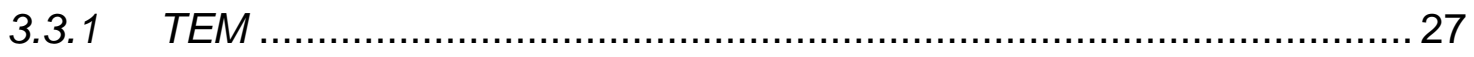

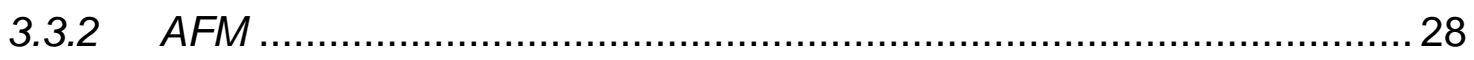

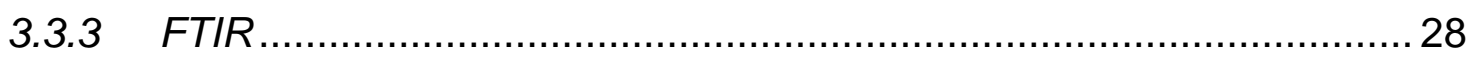

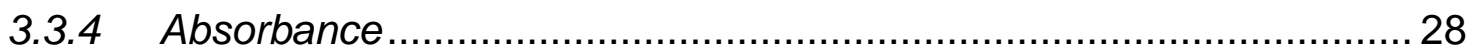

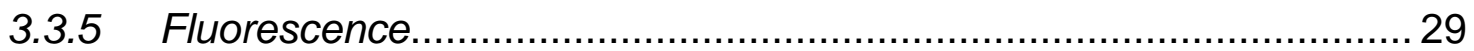

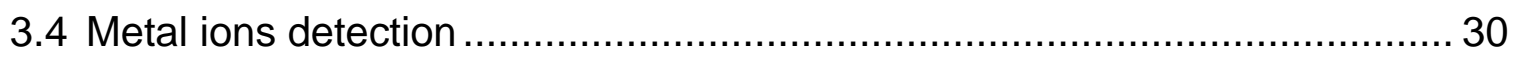

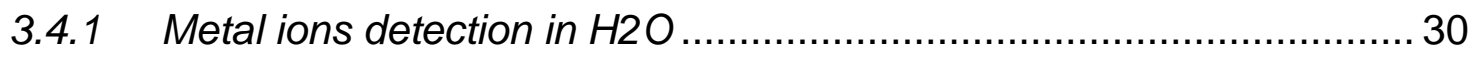

3.4.2 Metal ions detection in sol-gel thin films ........................................ 31

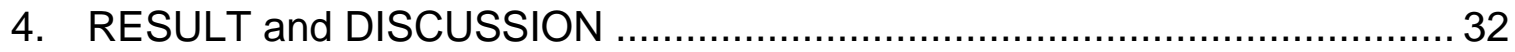

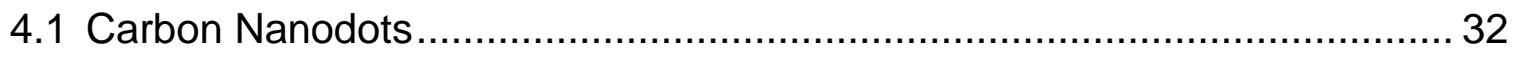

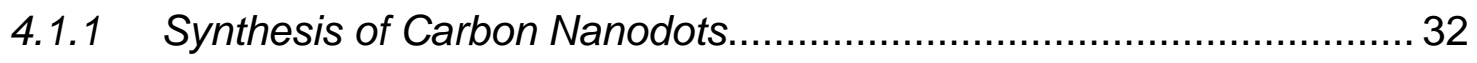

4.2 Carbon Nanodots Sol-Gel Thin Films ..................................................... 33

4.2.1 Sol-Gel Thin Film Preparation ........................................................... 33

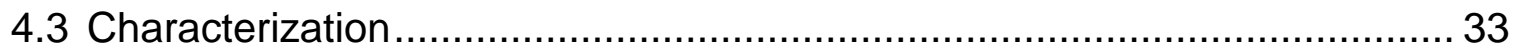

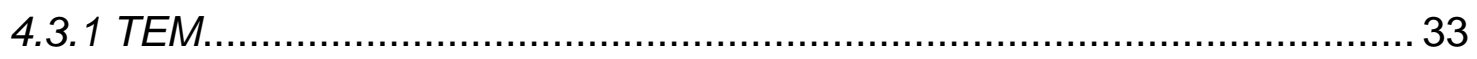

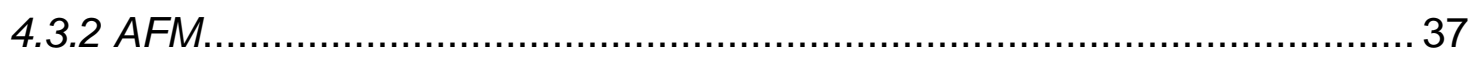

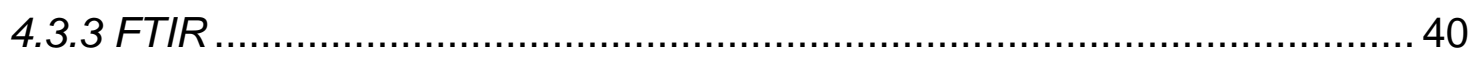




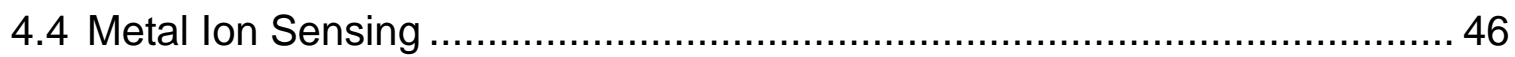

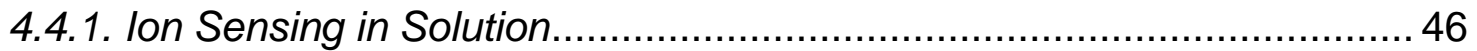

4.4.2 Ion Sensing in Sol-Gel Thin Films .................................................. 53

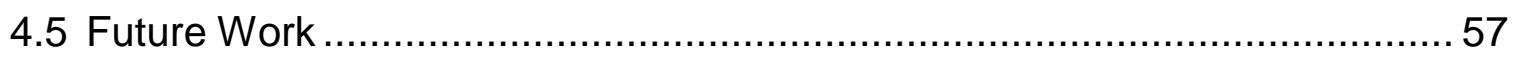

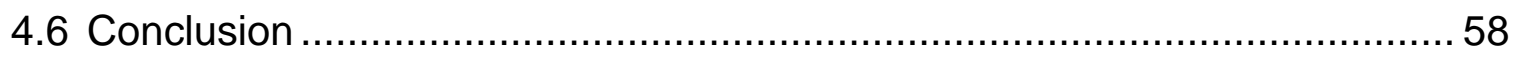

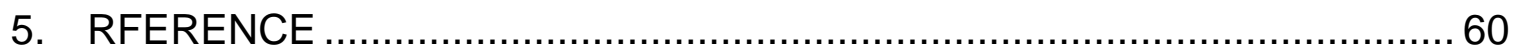




\section{TABLE OF FIGURES}

Figure 1.1 Jablonski Energy Diagram (from OLYMPUS Microscopy Resource

Center) which illustrates the electronic states and electrons transition in

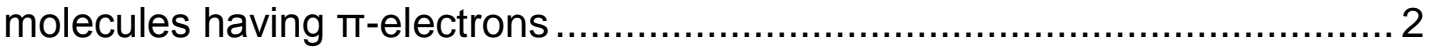

Figure 1.2 Scheme of condensation process of TEOS in sol-gel solution ${ }^{[11]} \ldots \ldots . .5$

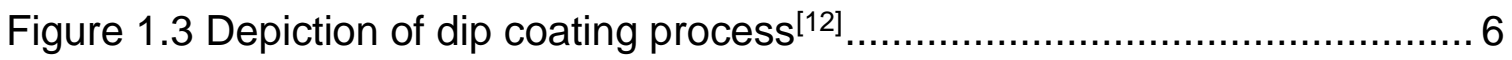

Figure 1.4 Depiction of spray coating with the utilization of an electro-pneumatic

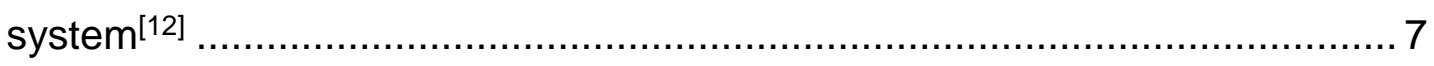

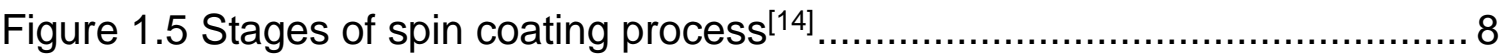

Figure 2.1 Depiction of the synthesis of multicolor C-dots on silica sphere

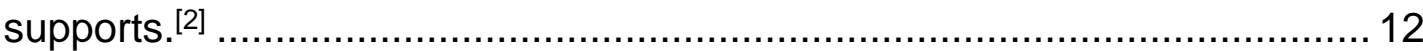

Figure 2.2 Dipolar molecules which attempt to align with electric field in high

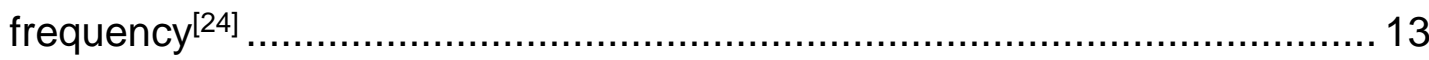

Figure 2.3 The movement of charged carriers (ions, electrons etc) followed the

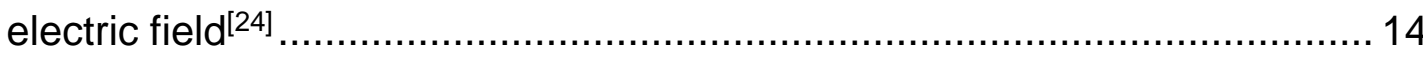

Figure 2.4 Two-photon luminescence image of human breast cancer MCF-7 cells with internalized C-dots passivated with PPEI-EI. ${ }^{[2]}$

Figure 2.5 EAC cells labeled with fluorescence C-dots. Bright field, UV, and blue light excitations were applied for images. The bottom row images correspond

to the control experiment, no C-dots were used. ${ }^{[15]}$ 16

Figure 2.6 Intravenous injection of C-dots in female DBA/1 mice : A) bright field,

B) as detected fluorescence (BI, bladder; Ur, urine), and C) color-coded 
images. The same order is used for the images of the dissected kidneys ( $A^{\prime}-$ $\left.C^{\prime}\right)$ and liver (A"-C"'). ${ }^{[2]}$

Figure 2.7 Responding fluorescence spectra (a) the CdSe@C-TPEA ratiometric probe and c) the single CQD-TPEA sensor without QDs as reference signals, exposed to the increasing concentration of $\mathrm{Cu}$ (II) ions (b) the dual-emission sensing solution (d) the single emission sensing solution. The inset of (a) shows the Stern-Volmer plot of relative emission as a function of the $\mathrm{Cu}$ (II)

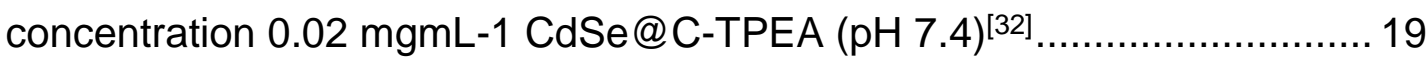

Figure 3.1 Image of CEM microwave used for the synthesis of C-dots ............. 25

Figure 3.2 Image of effendorf Centrifuge 5430/5430 R .................................... 25

Figure 3.3 6800 Spin Coater Series spin coater from Specialty Coating Systems

Figure 3.4 Tecnai $\mathrm{G}^{2}$ F30 Twin from FEl Company equipped with a $300 \mathrm{kV}$ field emission gun 27

Figure 3.5 Image of BioTek Powerwave HT Microplate Spectrophotometer, used for the detection UV-Vis absorption spectra of C-dots 29

Figure 3.6 Horiba Fluorolog Spectrafluorometer..... 30

Figure 4.1 Depiction of microwave-assisted synthesis of carbon nanodots from JEFFAMINE ${ }^{\circledR}$ T series which is examplified by the above structure, for JEFFAMINE ${ }^{\circledR}$ T3000, R=H, $n=0, x+y+z=50, M W=3000$; for JEFFAMINE ${ }^{\circledR}$ T5000, $R=H, n=0, x+y+z=50, M W=5000$ 
Figure 4.2 TEM image of C-dots (a),(c) T3000 C-dots (b),(d) T5000 C-dots, scale bar: (a),(b) $20 \mathrm{~nm}$; (c) (d) $10 \mathrm{~nm}$; and the corresponding particle size distribution of as prepared (e) T3000 C-dots, (f) T5000 C-dots. 36

Figure 4.3 AFM images for T3000 and T5000 C-dots with different magnification and the height analysis of representative (a)(c)(e) T3000 C-dots; (b)(d)(f) T5000 C-dots both dispersed in water 40

Figure 4.4 FTIR spectra of (a) T3000 C-dots (solid line) and TEOS, (b) T5000 Cdots (solid line) and TEOS 41

Figure 4.5 UV absorbance spectra and normalized emission spectra of C-dots prepared from JEFFAMINE® (a) T3000 $(\lambda \mathrm{ex}=375 \mathrm{~nm})$ and (b) T5000 $(\lambda \mathrm{ex}=365 \mathrm{~nm})$

Figure 4.6 Normalized fluorescence emission spectra with increasing longer excitation wave length (in $10 \mathrm{~nm}$ increments starting from $325 \mathrm{~nm}$ ) of C-dots in Ultrapure water produced from microwave irradiated a) JEFFAMINE $®$ T3000; b) JEFFAMINE® T5000. 46

Figure 4.7 Fluorescence response of T3000 and T5000 C-dots towards various metal ions, where each sample $(0.1 \mathrm{ml})$ was diluted in deionized water $(2.5$ $\mathrm{ml}$ ). The concentration of metal ions was $500 \mu \mathrm{M}$. Where $\mathrm{F}_{0}$ and $\mathrm{F}$ represents respectively the $\mathrm{FL}$ intensities of $\mathrm{C}$-dots in the absence and presence of $500 \mu \mathrm{M}$ metal ions in $\mathrm{H}_{2} \mathrm{O}$. Dash circle presented the $\mathrm{T} 3000 \mathrm{C}$ dots diluted with water to be the most sensitive and selective sensing system (illustrated in Figure 4.8). 48 
Figure 4.8 Fluorescence response of T5000 C-dots towards the addition of Fe (III) ions in water (concentration of Fe (III): 0, $500 \mu \mathrm{M}$ )

Figure 4.9 Fluorescence spectra of (a) T3000 C-dots and (b) T5000 C-dots responsing to the addition of $\mathrm{Fe}$ (III) ions in ultrapure water, and responding liner relationship between the concentrations of Fe (III) in ultrapure water and the relative fluorescence intensity of (c) T3000 C-dots and (d) T5000 C-dots, respectively 52

Figure 4.10 Scheme of condensation process of TEOS in sol-gel solution ${ }^{[11]} \ldots . .54$ Figure 4.11 Quenching effect of various metal ions on relative fluorescence intensity $\left(\mathrm{F}_{0} / \mathrm{F}\right)$ of sol-gel entrapped T3000 and T5000 C-dots in ultrapure water 


\section{LIST OF TABLES}

Table 4.1 List of parameters for the Stern-Volmer fits to the T3000 and T5000

emission peaks in water. 


\section{LIST OF ABBREVIATIONS}

Atomic force microscopy

AFM

Carbon nanodots

C-dots

Centimeters

$\mathrm{cm}$

Fluorescence emission intensity

FL

Fourier transform infrared spectroscopy

FTIR

grams

g

Microliters

$\mu L$

Micromolar

$\mu \mathrm{M}$

Milliliters

$\mathrm{mL}$

Millimolar

$\mathrm{mM}$

Molar

M

Normal

N

Ratio of fluorescence emission peak (initial)

$F_{0}$

Ratio of fluorescence emission peak (with ion addition) $\mathrm{F}$

Relative intensity

$\mathrm{F}_{0} / \mathrm{F}_{\text {, I }}$

Rotation per minutes

rpm

Sodium hydroxide

$\mathrm{NaOH}$

Tetraethyl orthosilicate

TEOS

Thin film

TF

Transmission electron microscopy

TEM 


\section{ABSTRACT}

Carbon nanodots were synthesized from JEFFAMINE ${ }^{\circledR}$ T3000 and T5000 as carbon source and with no passivation agent. The transparent organic compound changed to dark brown solution, followed by 15 heating-cooling cycles in a $300 \mathrm{~W}$ microwave for $45 \mathrm{~min} .1 \mathrm{~mL}$ deionized water was added to the cool down dark brown solution before centrifugation to extract C-dots from the solution. Finally, Cdots particles were well dispersed in the golden-yellow colored aqueous supernatant. The possible mechanism of C-dots formation may include four steps: dehydration, polymerization, pyrolysis and self-passivation. ${ }^{[1]}$ Without post surface passivation process, we have demonstrated that the one-step microwave assisted method is a simple, rapid and green synthetic route for the production of watersoluble C-dots with highly intense photoluminescence (PL). C-dots prepared through microwave pyrolysis were exhibited as uniform, spherical particles with small dimension and mono-dispersing in the solution in the TEM.

The prepared two types of C-dots displayed blue fluorescence under a UV-lamp excitation and exhibited emission peaks centered at $448 \mathrm{~nm}$ and $439 \mathrm{~nm}$ respectively. Moreover, our study showed that JEFFAMINE® T3000 and T5000 Cdots have the feature of $\lambda_{e x}$ dependent emission. The resulting photoluminescence of excited C-dots was spectrally broad in visible spectrum, with peak intensity wavelength ranging from $400 \mathrm{~nm}$ to $500 \mathrm{~nm}$ with a dependence on the excitation wavelength from 325 to $405 \mathrm{~nm}$. 
The non-passivated JEFFAMINE ${ }^{\circledR}$ T3000 and T5000 C-dots synthesized by the one-step microwave assisted route were also demonstrated to be useful as a fluorescence sensor with their remarkably selectivity and sensitivity of $\mathrm{Fe}$ (III) ions over other interfere metal ions in water. In this metal ions quenching study, the observed perfect linear relationship between the relative fluorescence intensity respond to the addition of $\mathrm{Fe}$ (III) ions (up to $500 \mu \mathrm{M}$ ) suggests that the JEFFAMINE ${ }^{\circledR}$ T3000 and T5000 C-dots could potentially be applied as excellent sensor materials for the detection of Fe (III) with high sensitivity and selectivity. Meanwhile, the sol-gel thin film immobilization of the JEFFAMINE® T3000 and T5000 C-dots was studied, however more intensive research effort to generate an applicable sol-gel immobilization of C-dots sensor is still required in the future.

Due to the advantages of low cost, easy preparation, high sensitivity and selectivity, T3000 and T5000 C-dots can be developed as a promising eco-friendly sensing probe for the detection of $\mathrm{Fe}$ (III) ions in a real sample in the near future. 


\section{INTRODUCTION}

\section{$1.1 \quad$ Fluorescence Mechanism}

Fluorescence is a form of luminescence which is the light emitted by a substance that absorbed light or electromagnetic radiation. The observed fluorescence of the studied carbon nanomaterial is a form of electronic phenomenon of molecules which contain conjugated $\pi$-electrons. When the molecules having conjugated $\pi-$ electrons absorb light radiation, the $\pi$-electrons firstly jump from the ground state (S0) to higher energy state (S1). To be capable of fluorescing, the majority of electrons go from the higher vibrational levels to the lowest one and are ready for radiative emission back to the ground state which resulting the emitted fluorescence. Due to the internal conversion, intersystem crossing and nonradiative relaxation, part of the energy loses during the electron transition procedure which explains the lower energy level of the emitted fluorescence compared to that of the absorbed light energy (named "Stroke Shift" or "Red Shift"). The principle of the fluorescence can be illustrated by the Jablonski Energy Diagram (Figure 1.1) which briefly explains the procedure of the electrons transition between different energy levels after absorbing certain light. The photoluminescence of the C-dots is one of the most fascinating features but not totally understood. The observed excitation wavelength dependent emission of C- 
dots may be the result of the optical selection of various nanoparticle size and/or the emissive traps on the surface of the C-dots. ${ }^{[2]}$

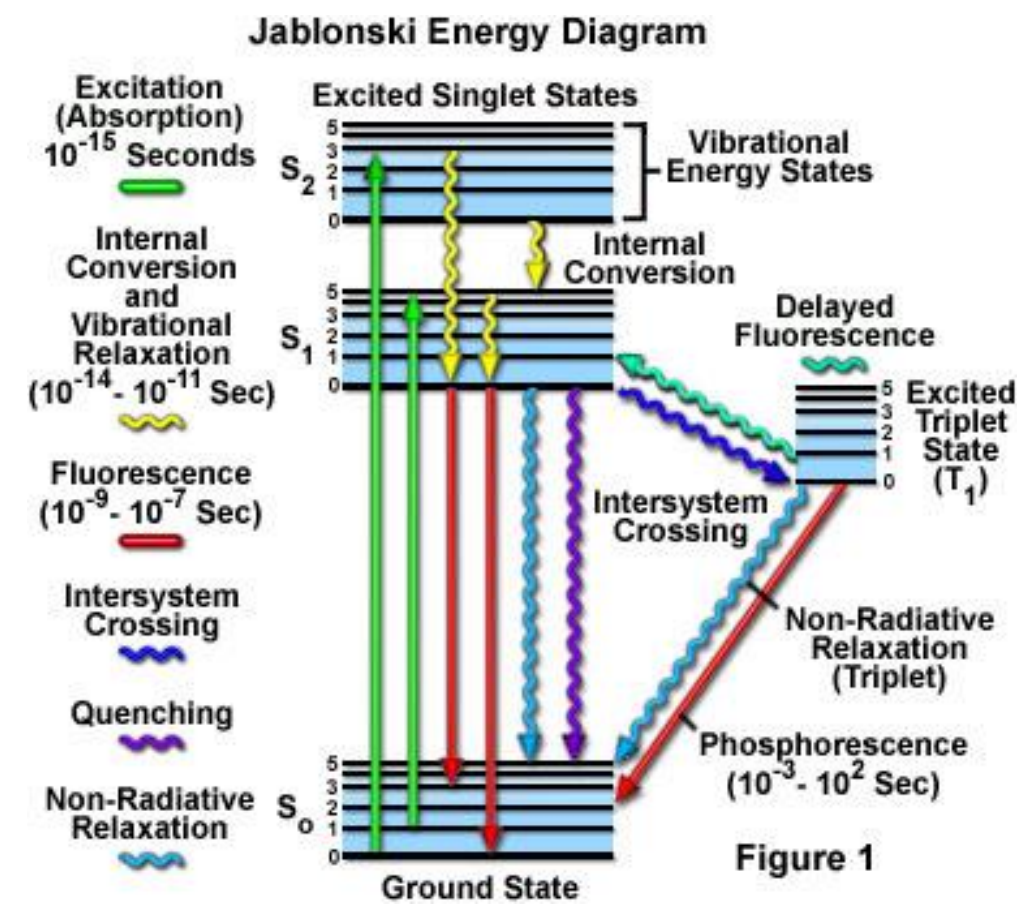

Figure 1.1 Jablonski Energy Diagram (from OLYMPUS Microscopy Resource Center) which illustrates the electronic states and electrons transition in molecules having $\pi$ electrons

\subsection{Carbon Nanodots (C-dots)}

In the past few years, the carbon nanodots, so called C-dots, a kind of novel nanomaterial, that are inspiring increasing attention among researchers throughout the world. C-dots are defined as a form of nanoparticles with features of discrete, quasi-spherical and size usually below $10 \mathrm{~nm} \cdot{ }^{[2]}$ Although semiconductor quantum dots (QDs) can be functionalized with various bioactive 
moieties, allowing them capable of drug targeting and in vivo biomedical imaging, some QDs have been demonstrated to be hazarded to human health and environment. ${ }^{[3]}$ Carbon nanodots will be one of the ideal eco-friendly potential candidates for QDs due to their unique advantage of low cytotoxicity, thus imparting them with favorable property of biocompatibility. The application of Cdots as biological labeling, biomedicine, optoelectronic devices and sensors has been widely studied due to their properties of excitation wavelength dependent photoluminescence, chemical and photo stability, and low cost preparation steps with high efficiency, etc. ${ }^{[4]}$

\subsection{Sol-Gel Thin Film}

Sol-gel is a process used for the preparation of inorganic glasses at room temperature which was developed in the $19^{\text {th }}$ century. ${ }^{[5]}$ It was firstly completely investigated by Avnir et al. in 1980s who successfully embedded Rhodamine 6G in silica gel glass. ${ }^{[6]}$ The sol-gel thin film method is applied to investigate the fluorescence properties of C-dots, where a large integrated gel network was polymerized from tetraethyl orthosilicate (TEOS), a widely used chemical precursor in sol-gel synthesis. ${ }^{[7]}$ Two main reactions are involved in the preparation of the sol-gel process. The first step is hydrolysis where TEOS firstly react with water and are catalyzed by acid which help increasing the hydrolysis rate. In the second step condensation, tons of siloxane bonds which are formed from the 
polymerization of water production are provided from the hydrolysis step, reactions are shown ${ }^{[5,8]}$ :

$$
\begin{gathered}
\mathrm{Si}(\mathrm{OR})+4 \mathrm{H}_{2} \mathrm{O} \stackrel{\mathrm{ACID}}{\longrightarrow} \mathrm{Si}(\mathrm{OH})_{4}+4 \mathrm{ROH} \\
n \mathrm{Si}(\mathrm{OH})_{4} \rightarrow n \mathrm{SiO}_{2}+2 \mathrm{nH}_{2} \mathrm{O}
\end{gathered}
$$

There are typically five main steps involved in the development of the sol-gel system: alkoxide sol formation, gelation, aging, drying and densification. Hydrolysis is introduced by the addition of water in acidic, neutral or basic conditions. An SN2-type reaction leads to the formation of a silanol moiety with an acid catalyst. The followed up condensation reaction brings the silanol moieties aggregating together and create tons of siloxane linkages, as illustrated in the Figure 1.2, finally allows a sol formed from these siloxane particles. A sufficient number of linkages are provided from the condensation process before the gelation can transpire in the system. ${ }^{[9]}$ In the gelation step, a two-phase system contains amorphous particles and an interstitial liquid phase is irreversibly converted from the singles phase liquid system. After the completion of the gelation step, an aging process is occurred to enlarge the cross-linking degree in the system. The liquid phase is removed from the pores of the amorphous particles in the followed drying step, which eventually resulting the sol-gel shrinking and the pores disintegrating, the last steps, that keeps the sol-gel to be denser. ${ }^{[5]}$ The solgel technique has been applied to produce a variety of sample types, for example thin films, fibers, bulk monoliths, and powders etc. ${ }^{[5,10]}$ 


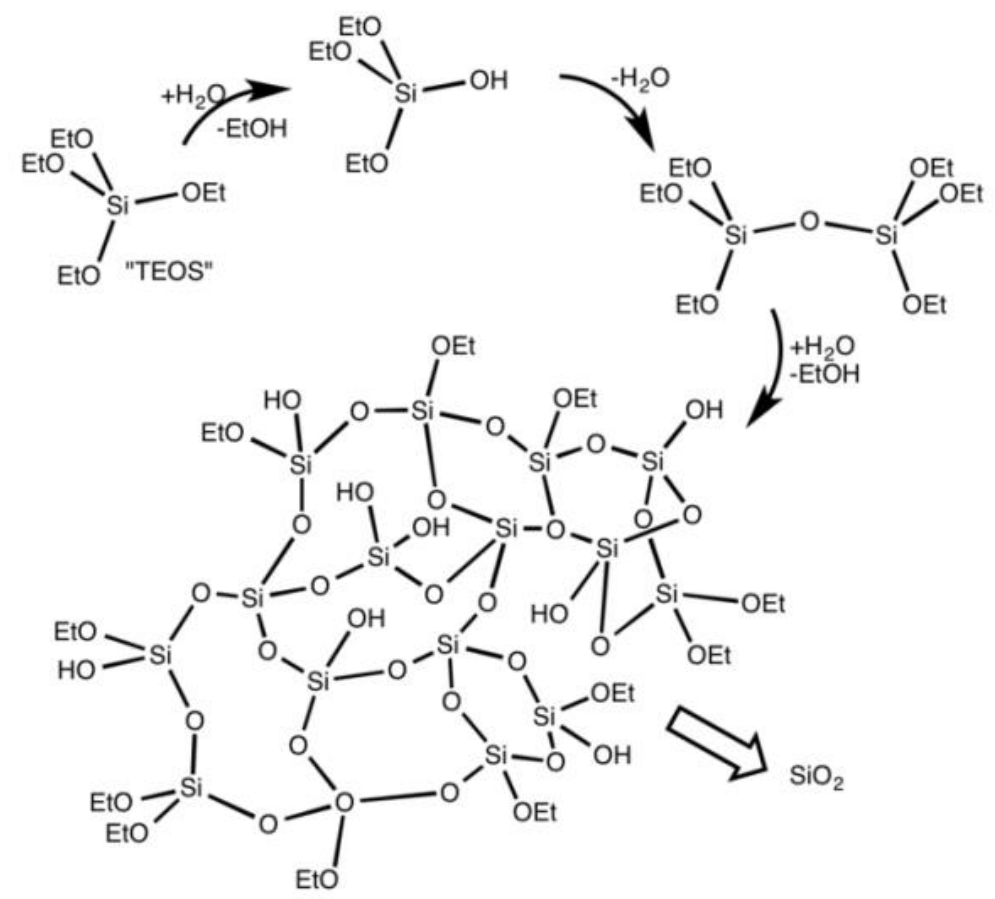

Figure 1.2 Scheme of condensation process of TEOS in sol-gel solution ${ }^{[11]}$

The sol-gel technology has been widely studied for the application of chemical optical sensors, biosensors and electronic coatings thanks for its simplicity and versatility. The reported optical sensors can be divided according to their main application such as the quantity and/or quantify $\mathrm{pH}$, gases, ionic species and solvents etc. To meet the increasing need for stable, reliable biosensors for the application in food industry, diagnostics, in vivo monitoring, environmental and health control, sol-gel biosensors have attracted many efforts and experiencing a rapid growth in the past few years. Sol-gel porous structure has the advantages of thermally and mechanically stable, resist of photo degradation, being transparent to wavelength longer than $250 \mathrm{~nm}$. They are available in various configurations including thin films, fibers, monoliths and powders, with high compatibility to other materials. Thin film sol-gel glasses are defined as those with thickness below $1 \mu \mathrm{m}$, 
which means that tiny little amount of precursors and functional molecules are required for the coating. They are usually transparent, fast-responding, with higher mechanical resistance and demonstrated less cracking compared to its cousin monoliths. Also, thin film sol-gel glasses may probably allow the option of making multi-layer configuration and miniaturization. ${ }^{[10 a]}$

Sol-gel thin film can be prepared through three main approaches of dip coating, spin coating and spray coating. ${ }^{[10 a]}$ Dip coating process consists of five steps: immersion, starting, deposition, drainage and evaporation, which are illustrated in the Figure 1.3 below. In the immersion steps, the substrate is firstly immersed into the coating material for deposition. The substrate starts being pulled out after a determinate period of time for immersion. The deposition is occurred during the withdrawal, which should be carried out at a constant rate to form a uniform thin film, and at an appropriate speed that determine the thin film thickness. The excess of material is drained from the substrate surface in the drainage step. In the evaporation steps, the coating is formed with the evaporation of the solvent material from the deposited thin film on the substrate. ${ }^{[12]}$

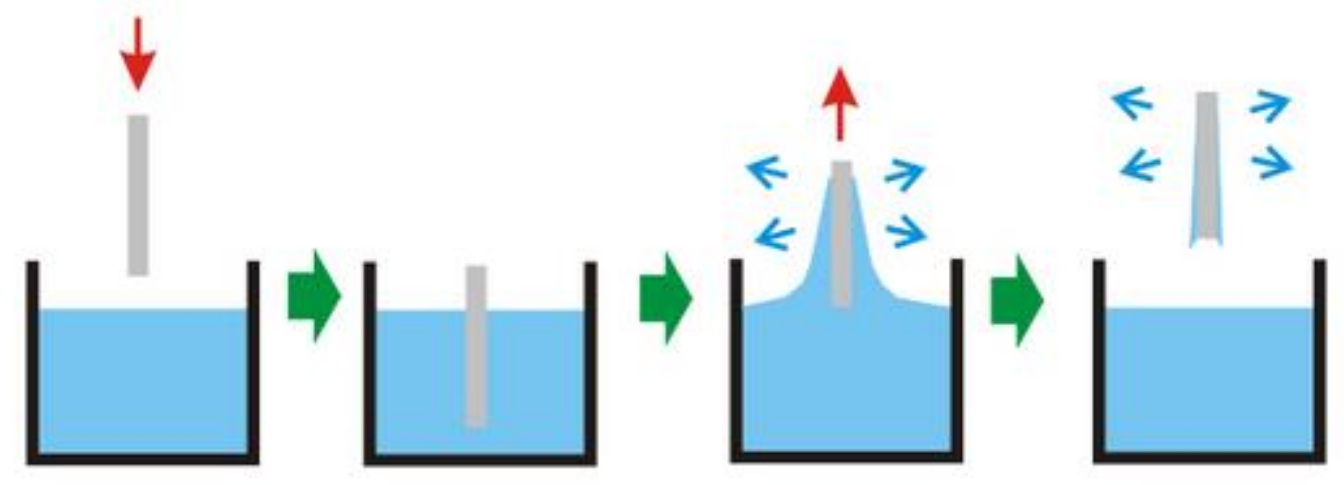

Figure 1.3 Depiction of dip coating process ${ }^{[12]}$ 
The spray coating is a coating process in which the substrate is coated with liquid material via spraying. Usually, an electro-pneumatic system is applied to keep the activation and deactivation of the nozzles or substrates in control as shown in the Figure 1.4..$^{[12]}$

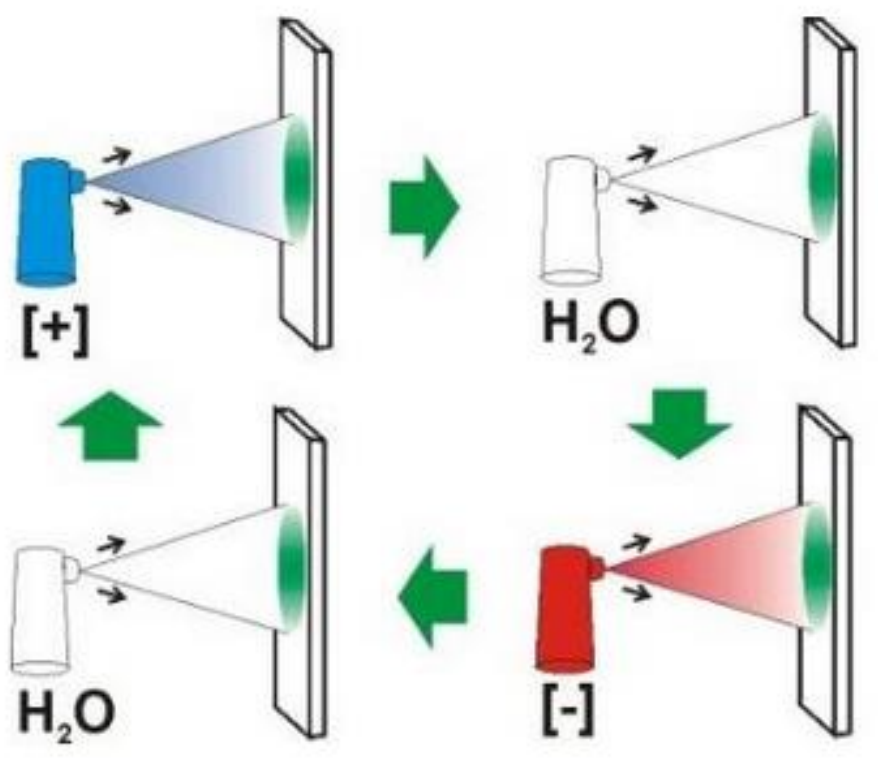

Figure 1.4 Depiction of spray coating with the utilization of an electro-pneumatic system ${ }^{[12]}$

Spin Coating is a coating technique that has been used for performing thin film since several decades ago. The two forces, centrifugal force and viscous force, are the main elements that determine the coating quality. The spin coating process can be divided into four stages, firstly the coating material is dispensed onto the substrate, with the increasing spinning speed, and the material is well spread across the substrate. Then, the higher speed is applied before the film is eventually 
formed after the edge bead is removed by the surface tension at the edge of the substrate (Figure 1.5). ${ }^{[13]}$

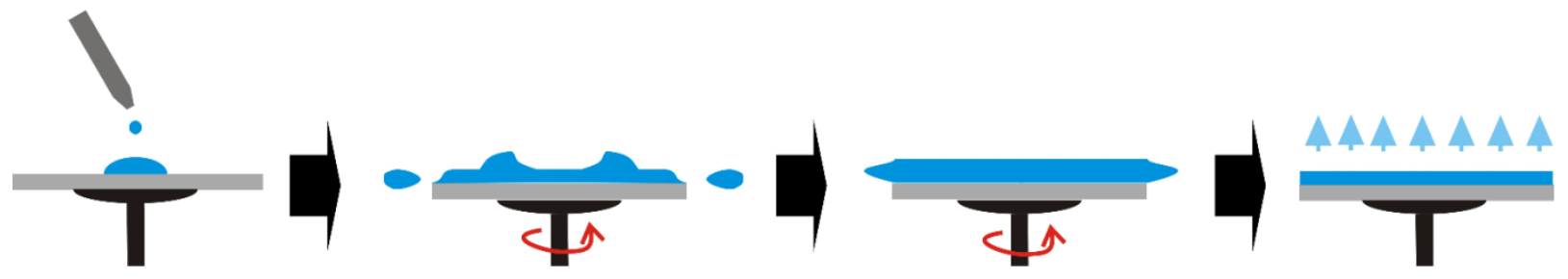

Figure 1.5 Stages of spin coating process ${ }^{[14]}$ 


\section{LITERATURE REVIEW}

\subsection{Carbon Nanodots}

In the past few years, the carbon nanodots (C-dots), so called C-dots, a kind of novel nanomaterial, are inspiring increasing attention among researchers throughout the world. Although semiconductor quantum dots (QDs) can be functionalized with various bioactive moieties, allowing them capable of drug targeting and in vivo biomedical imaging, some QDs have been demonstrated to be hazarded to human health and environment. Carbon nanodots will be one of the ideal eco-friendly potential candidates for QDs due to their unique advantage of low cytotoxicity, thus imparting them with favorable property of biocompatibility. The application of C-dots as biological labeling, biomedicine, optoelectronic devices and sensors has been widely studied due to their properties of excitation wavelength dependent photoluminescence, chemical and photo stability, and low cost preparation steps with high efficiency, etc. For example, Ray et al. reported the application of non-passivated C-dots for boosting the bioimaging by using Ehrlich ascites carcinoma cells. ${ }^{[15]}$

There have been reported that various routes can be applied to synthesize C-dots over the past few decades. Xu et al. firstly discovered this nascent carbonaceous material serendipitously when they were conducting gel electrophoresis to purify the single-walled carbon nanotubes (SWCNTs) which were synthesized by arc- 
discharge method. ${ }^{[16]}$ There generally two high-purity graphite electrodes are involved in the arc discharge technique acting as the anode and the cathode. A carbon rod will be build up at the cathode side when the arc is discharging for a while. SWCNTs are produced through this method followed by purification. However, an unexpected carbon nanomaterial with strong photoluminescence was also isolated as the third electrophoretic bands apart from the SWCNTs and short tubular carbons, which indicated the very beginning of the research on the referred carbon nanodots in 2004. Arc-discharge and developed laser-ablation and electrochemical synthesis are classified in the top-down approach family where Carbon nanodots are broken off from a larger carbon structure. Sun et al. were able to produce strong photoluminescence C-dots via laser ablation with argon as carrier gas and in the presence of water vapor. They started with hot-pressing a mixture of graphite powder and cement, stepwise baking, curing and annealing were performed in argon flow. ${ }^{[17]}$ The carbon target was ablated with a Q-switched Nd: YAG laser $(1064 \mathrm{~nm} 10 \mathrm{~Hz})$ in a flow of argon gas and water vapor at $900 \stackrel{\circ}{\mathrm{C}}$ and $75 \mathrm{kPa} .{ }^{[17]}$ Electro-oxidation of graphite for carbon nanocrystals was reported by Zhao's group, the system consisted of a graphite column electrode and a saturated calomel electrode with a Pt wire counter electrode soaking in $0.1 \mathrm{M}$ $\mathrm{NaH}_{2} \mathrm{PO}_{4}$ aqueous solution serving as the supporting electrolyte. ${ }^{[18]}$ To remove the non-fluorescent deposit, the electrolyte was centrifuged for 30 min followed by being ultrafiltered through centrifugal filter devices. Carbon nanocrystals emitted blue and yellow fluorescence were produced. ${ }^{[18]}$ 
Bottom-up methods in which C-dots are obtained from molecular precursors include combustion/thermal routes, supported routes and the studied microwave assisted method. Candle soot or natural gas burners are usually employed to prepare C-dots as typical way of combustion/thermal method which was followed by Ray and Chen's group. Ray et al. collected the candle soot from burning candle and refluxed it for $12 \mathrm{~h}$ with $5 \mathrm{M} \mathrm{HNO}_{3}$ to obtain the C-dots suspending solution. The C-dots particles were precipitated and then collected after several runs of centrifugation at high speed. ${ }^{[19]}$ Chen's group purified C-dots from carbon source of natural gas burner. PL exhibiting C-dots of $4.86 \pm 0.6 \mathrm{~nm}$ in diameter were obtained from soot being refluxed in $5 \mathrm{M} \mathrm{HNO}_{3}$ for $12 \mathrm{~h}$, centrifuged and dialyzed. No extra surface passivation was performed, however it was undetermined if there existed excitation wavelength dependent behavior on the as prepared C-dots. ${ }^{[20]}$ Another bottom-up synthetic strategy is so called supported route, the C-dots are blocking on the supports where the nanoparticles agglomerate during high temperature treatment. ${ }^{[2]} \mathrm{Li}$ and co-workers' had carbon precursor polymerized on the shell of the supports, which were surfactant-modified silica $\left(\mathrm{F} 127 / \mathrm{SiO}_{2}\right.$ composites) sphere. The Figure 2.1 represents the procedure of supported routes. Heating of $900{ }^{\circ} \mathrm{C}$ in argon for $2 \mathrm{~h}$ was performed on the spheres to obtain the $\mathrm{C}$ dots/SiO2 composites. C-dots of $1.5-2.5 \mathrm{~nm}$ in size were released followed by removing silica spheres. C-dots were then refluxed in the in $3 \mathrm{M} \mathrm{HNO}_{3}$ for $24 \mathrm{~h}$ followed by neutralization and dialysis for surface passivation. Yet either the requirement of stringent reaction system, expensive carbon precursors or the large 
amount of strong acid that limit the further application of these synthesis routes according to the environment and economic concern. ${ }^{[21]}$

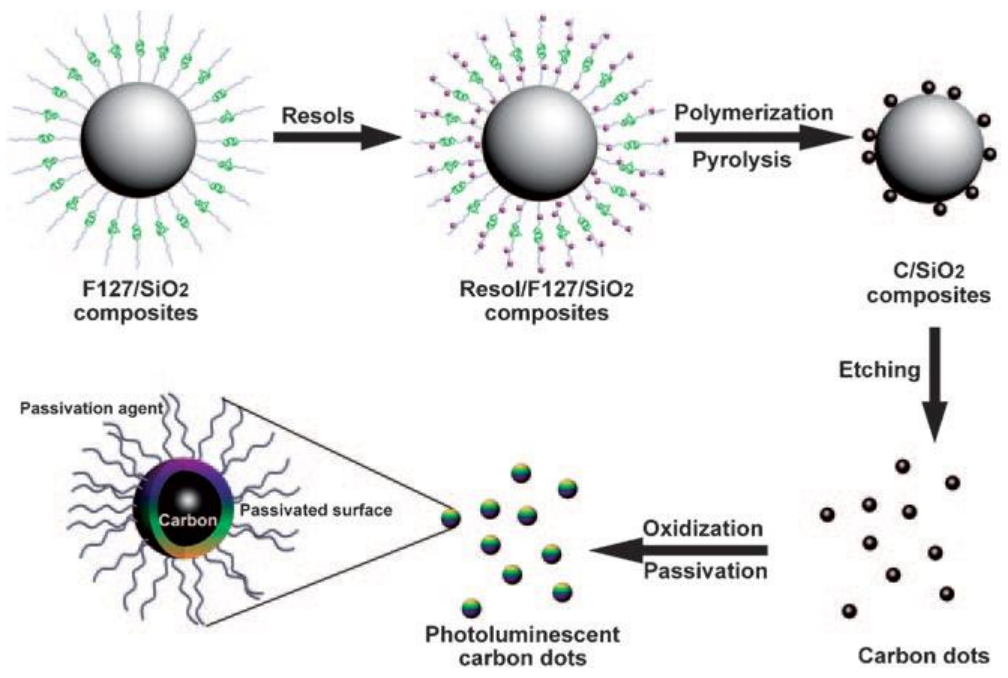

Figure 2.1 Depiction of the synthesis of multicolor C-dots on silica sphere supports. ${ }^{[2]}$

More recently, the rapid expansion of publications on microwave-assisted polymerization indicate the popularity of the novel synthesis approaches in chemistry. ${ }^{[22]}$ Reagents and solvents are provided with a rapid and uniform heating by microwave which accelerates the reaction, usually leading to monodispersing smaller nanoparticles with narrower size distribution. ${ }^{[23]}$ Furthermore, phenomenon superheating resulted from microwave irradiation is able to raise the boiling points of solvents up to $26{ }^{\circ} \mathrm{C}$ higher than the conventional values, especially in pure solvents, the superheating can be kept as long as the microwave irradiation is performed. ${ }^{[24]}$ Compared with the conventional heater such as oil baths, sand baths and heating jacket, the microwave dielectric heating effect allows the microwave energy directly to be applied on the reactants and solvents rather than on the reacting vessel, thus increasing the temperature rapidly and 
homogeneously. Dipolar polarization and electrical conduction are the two underlying mechanisms that are responsible for the microwave dielectric heating. [24]
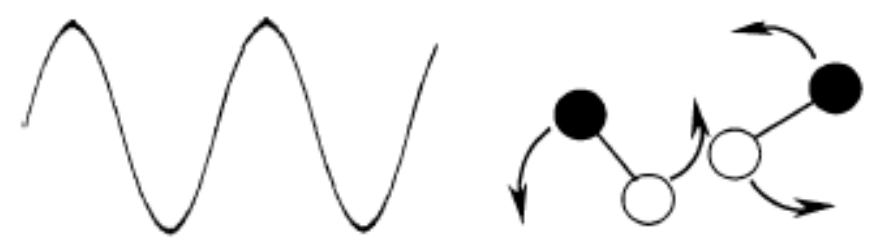

Figure 2.2 Dipolar molecules which attempt to align with electric field in high frequency ${ }^{[2]}$

Microwave for chemical reaction applies a high electric frequency field to the dipole molecules, makes the dipole rotating and aligning to the electrical field, during which large amount of heat is generated by these rotating dipoles and therefore accelerates the reaction. Figure $\mathbf{2 . 2}$ models the rotation of the dipole molecules in the high frequency electric field provided by microwave. The other electrical conduction provides much stronger interaction on the reaction than the dipolar mechanism does due to its higher heat generation capacity. Figure 2.3 illustrates the collision of charged carriers such as ions, electrons etc. in the same alignment of the applied electric field. The ions, electrons in reactant will follow with the movement of the solution, increase collision rate, result the generation of heat converted from the kinetic energy. ${ }^{[24-25]}$ 


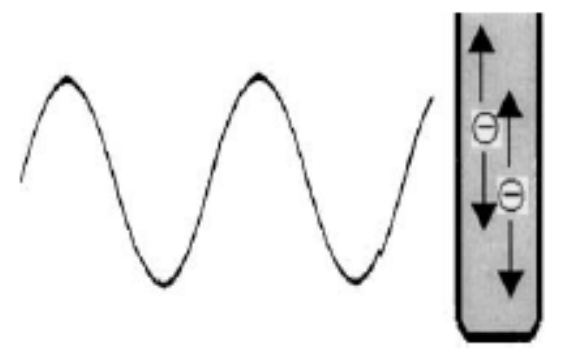

Figure 2.3 The movement of charged carriers (ions, electrons etc) followed the electric field $^{[24]}$

Zhu et al. reported a facial one-step MW pyrolysis approach to synthesize carbon nanoparticles passivated with $\mathrm{PEG}_{200}$ in a $500 \mathrm{~W}$ microwave for minutes. ${ }^{[26]}$ Peng and Travas-Sejdic introduced an aqueous solution route for C-dots preparation from dehydrated carbohydrates and applied amine-terminated compounds as surface passivation reagent to enhance the fluorescence luminescence of the prepared C-dots. ${ }^{[27]}$ More recently, Elaheh Farjami and his co-workers successfully produced strong PL C-dots with average size of $6 \mathrm{~nm}$ via a $450 \mathrm{~W}$ a microwave by using ionic liquid as carbon source. FTIR spectra revealed the existence of large numbers of $\mathrm{C}-\mathrm{OH}$ and $-\mathrm{OH}$ groups on the surface of the nonpassivated $\mathrm{C}$-dots which impacted them with high water solubility. ${ }^{[28]}$

Due to their advantages of unique tunable optical and eco-friendly properties, Cdots are well developed to be attractive candidates for the application in targeting and bioimaging. Cao et al. demonstrated firstly the bioimaging capabilities of Cdots passivated with PPEI-EI using human breast cancer MCF-7 cells in two photon luminescence microscopy. The targeted cells emitted bright luminescence 
in fluorescence microscope after being incubated with C-dots for 2 hours at 378 ${ }^{\circ} \mathrm{C}$ and washed to remove residual $\mathrm{C}$-dots in cellular (Figure 2.4). [4a]
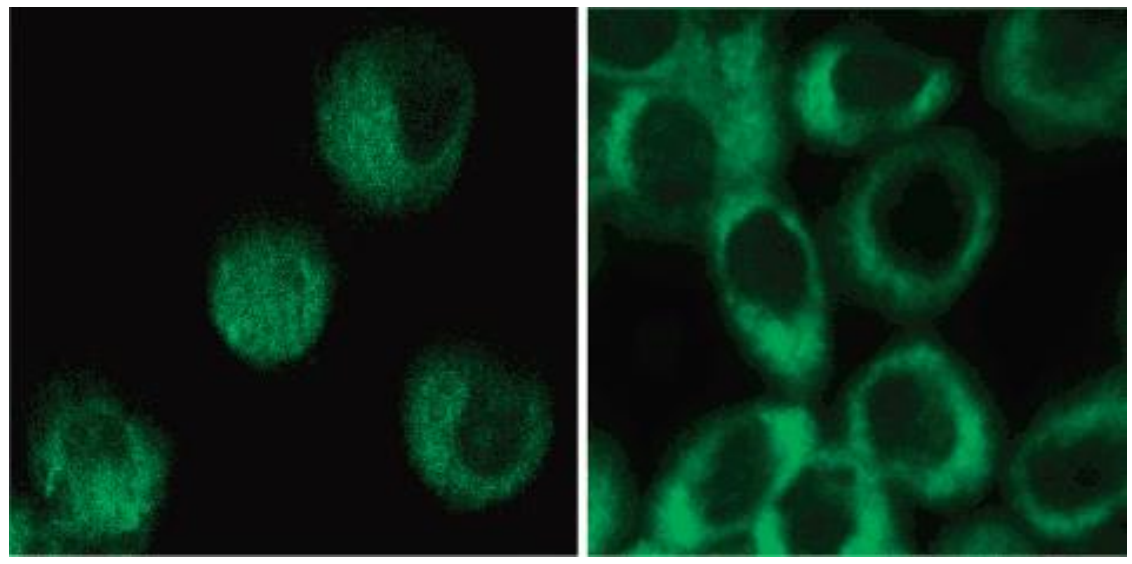

Figure 2.4 Two-photon luminescence image of human breast cancer MCF-7 cells with internalized C-dots passivated with PPEI-El. ${ }^{[2]}$

Ray et al. used C-dots labeling Ehrlich ascites carcinoma cells (EACs) in aqueous solution and investigated the bioimaging under an Olympus IX71 fluorescence microscope equipped with a digital camera. Figure 2.5 showed that the unpassivated C-dots penetrated into the cells, enabled the cells exhibited bright blue-green photo luminescence under UV excitation. Yet luminescence was barely observed when the C-dots were not involved as indicated by the control experiment. ${ }^{[15]}$ 

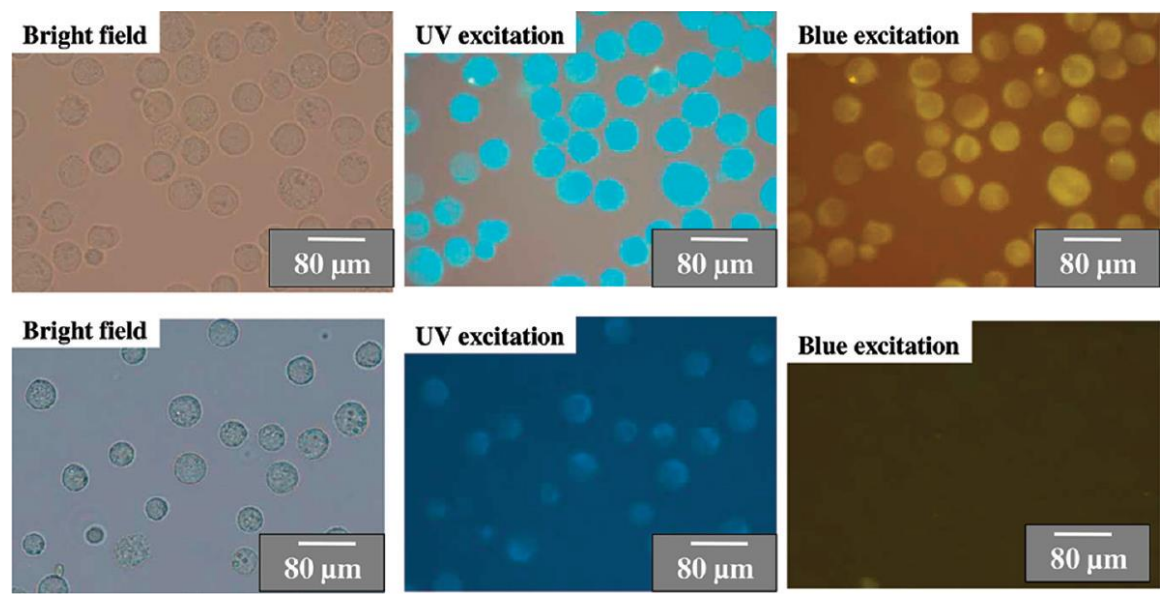

Figure 2.5 EAC cells labeled with fluorescence C-dots. Bright field, UV, and blue light excitations were applied for images. The bottom row images correspond to the control experiment, no C-dots were used. ${ }^{[15]}$

C-dots are ideal in vivo fluorescent contrast agents since they are bright, nontoxic, biocompatible, and stable against photobleaching. Sun and his co-workers reported a in vivo studies of C-dots as optical imaging agent, which were synthesized by laser ablation. The PL emission of the injected C-dots can be detected in bladder area. The C-dots showed the potential for targeting the organs like kidney and liver in female DBA/1 mice as present in the Figure 2.6. ${ }^{[2]}$ 


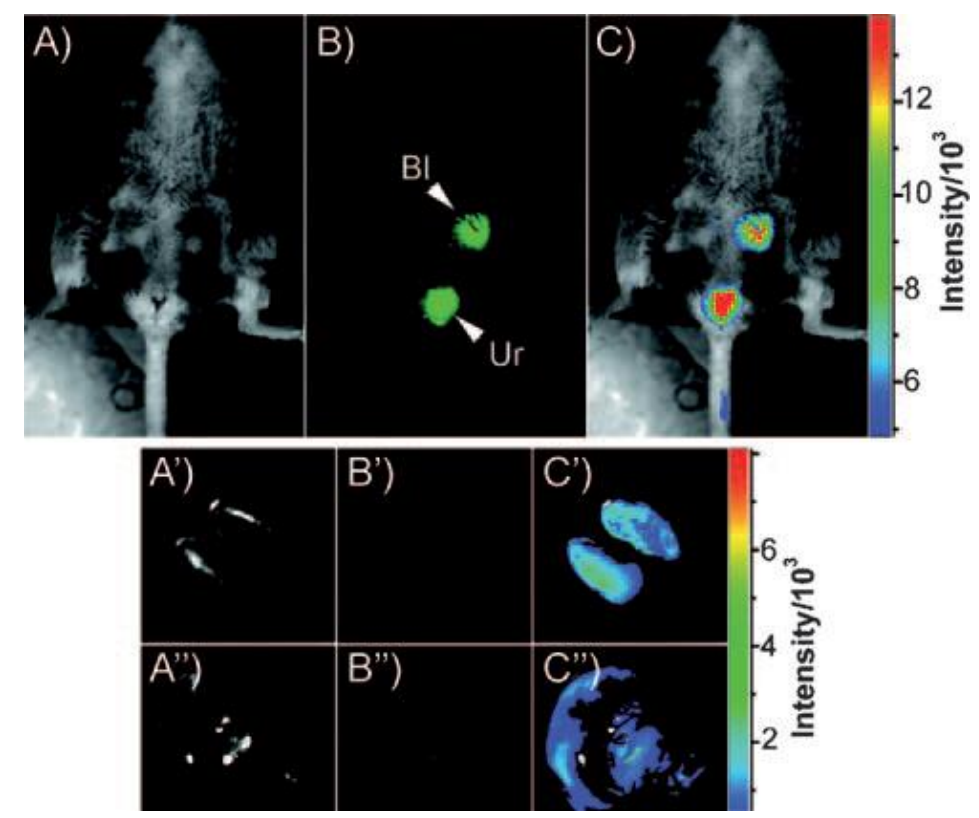

Figure 2.6 Intravenous injection of C-dots in female DBA/1 mice : A) bright field, B) as detected fluorescence (Bl, bladder; Ur, urine), and C) color-coded images. The same order is used for the images of the dissected kidneys $\left(A^{\prime}-C^{\prime}\right)$ and liver $\left(A^{\prime \prime}-C^{\prime \prime}\right) \cdot{ }^{[2]}$

PL C-dots have been investigated for their application as sensor in environmental areas. Fluorescence quenching has been proven to be one of the most effective analysis approaches applied to quantify and quality various metal ions including $\mathrm{Sn}$ (II), $\mathrm{Hg}$ (II), and $\mathrm{Cu}$ (II). Sn (II) ions is a type of heavy metal and regarded as one of environmental pollutants in natural water. Sing Muk $\mathrm{Ng}$ and the co-workers demonstrated the ability of fluorescent C-dots to detect Sn (II) in water. The C-dots fluorescence was significantly quenched by Sn (II) ions concentrations up to 4 mM. ${ }^{[29]}$ Zhou et al. used unmodified carbon nanodots to detect $\mathrm{Hg}$ (II) ions in TrisHCL buffer at $\mathrm{pH}$ 8.5. ${ }^{[30]}$

Goncalves et al. described a fast, reversible and stable sol-gel immobilized carbon nanodots sensor system for detection of $\mathrm{Hg}$ (II) ions in water. ${ }^{[31]}$ Zhu et al. designed 
a dual-emission fluorescence probe for detection of intercellular $\mathrm{Cu}$ (II) ions based on C-dots hybridized with CdSe/ZnS QDs (CdSe@C-TPEA), which were served as reference signals in the sensing system. As illustrated in Figure 2.7 , the quenching fluorescence intensity of reactive C-dots was much more distinguished by naked eye in the presence of the red background emission from the CdSe/ZnS QDs (Figure 2.7b) compared with the one in the absence of the reference red emission (Figure 2.7d). ${ }^{[32]}$

Moreover, the highly luminescent carbon nanodots have been proven their own advantages as sensors in biological areas. Niu and co-workers firstly developed an eco-friendly molecularly imprinted silica nanospheres embedded carbon nanodots (CDs@MIP) to detect dopamine in aqueous solution, whose fluorescence intensity decreased linearly with the increasing dopamine concentrations. And the CDs@MIP sensing system was successfully applied in human urine samples with good reliability. ${ }^{[33]}$ 
a)

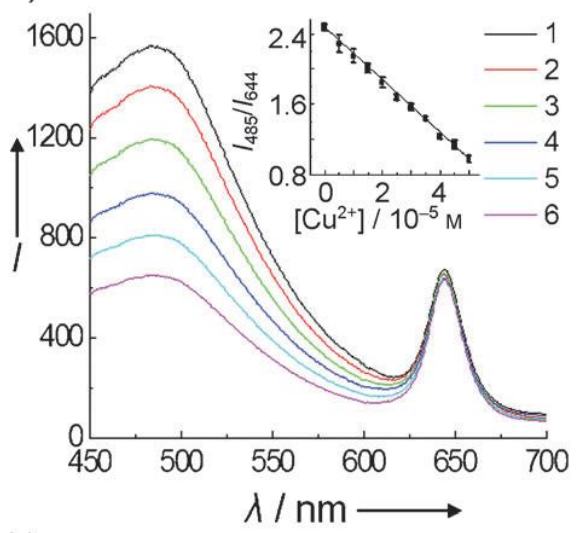

b)

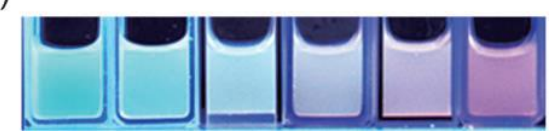

c)

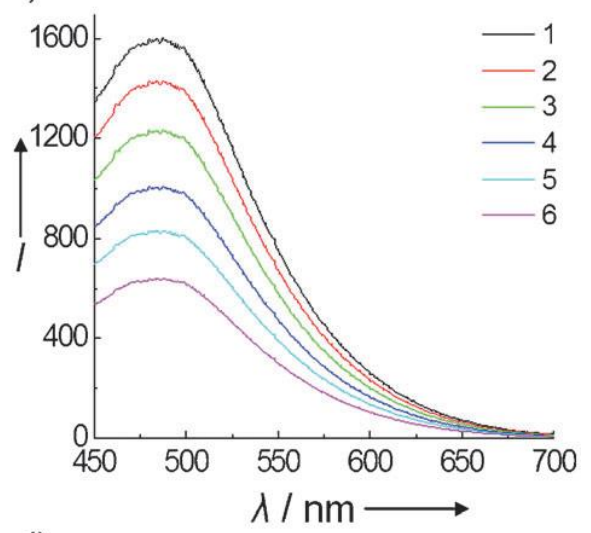

d)

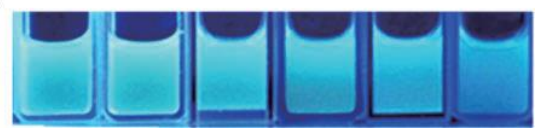

Figure 2.7 Responding fluorescence spectra (a) the CdSe@C-TPEA ratiometric probe and c) the single CQD-TPEA sensor without QDs as reference signals, exposed to the increasing concentration of $\mathrm{Cu}$ (II) ions (b) the dual-emission sensing solution (d) the single emission sensing solution. The inset of (a) shows the Stern-Volmer plot of relative emission as a function of the $\mathrm{Cu}$ (II) concentration 0.02 mgmL-1 CdSe@C-TPEA (pH $7.4)^{[32]}$

\subsection{Sol-Gel Thin Film}

The sol-gel immobilization of dyes and molecules for the application as sensors has been developed to detect ions and $\mathrm{pH}$ in the past decades. Kuselman et al. developed an effective sensing system for Fe (II) ions targeting by using ophenanthroline doped sol-gel silica powder in capillary tubes. ${ }^{[34]}$ Wolfbeis et al. investigated the application of a sol-gel immobilized a mixture of two isomers (5and 6- carboxynaphthofluorescein CNF) as a LED compatible fluorimetic pH sensor in the range 6-9. ${ }^{[35]}$ Another sensing system was developed based on the 
fluorescence properties of the sol-gel immobilized dyes and nanomaterial inside for the detection of various ions. Wallington et al. used Morin immobilized in sol-

gel for tracking $\mathrm{Al}$ (III) ions through the responding fluorescence intensity quenching. ${ }^{[36]}$ Flora et al. studied the sensing ability of a sol-gel entrapped parvalbumin probe to detecting $\mathrm{Cu}$ (II) utilizing a fluorescent allosteric signal transduction strategy. ${ }^{[37]}$ The various entrapped dyes and molecules in sol-gel allow sol-gel system possibility of being effective, biocompatible and eco-friendly sensor for environmental and biomedical purposes.

\subsection{Motivation}

Fluorescent semiconductor quantum dots (QDs) have a bunch of invaluable benefits and are excellent materials for biosensing, labeling and bioimaging, however they may be risky not only to human body but to the environment as well, which is a growing concern and inspiring more and more effects in scientists throughout the world. Carbon nanodots, act as new type of eco-friendly alternatives to the QDs, are arising intensive attention. Their low or even noncytotoxicity are demonstrated by being injected into live mice. Both biocompatibility and biosafety are far more than satisfactory.

Microwave route has been demonstrated to be a green and effective synthesis route for C-dots production and highly studied in the past few years, but the requirement of high power, external surface passivation reagents and concentrated strong acid limit the further application in industry. In our present 
work we successfully synthesized water-soluble carbon nanodots via the microwave-assisted method using JEFFAMINE $^{\circledR}$ T3000 and T5000 as the precursors. Without post surface passivation process, we have demonstrated that the one-step microwave-assisted method is a simple, rapid and green synthetic route for the production of water-soluble C-dots with high intense photoluminescence.

In addition to the excellent fluorescence activity, the as-prepared carbon nanodots are also envisioned to be of great sensitivity and selectivity in tracking and detecting Fe (III) ions in water. ${ }^{[38]}$ Iron is one of the essential elements for human body, plays a vital role in the metabolism of oxygen and electron transfer, which is especially important for growing children, childbearing and menstruating women. However, excess iron accumulation in hepatocytes and hemochromatosis will be one of the major reasons that increase the risk of cirrhosis and hepatocellular carcinom, reported by patients with hemochromatosis, some of whom even die because of the biological. ${ }^{[39]}$ Herein, it is desirable to develop a reliable, sensitive and fast analytical method to qualify and quantify the level of iron presenting in the environment and human body. Wang et al. introduced a Fe (III) ions detection probe by track the quenching effect on the fluorescence of the graphene oxide sheets modified by periodic acid. ${ }^{[40]}$ Wang et al. reported synthesized Rhodamine modified $\mathrm{Fe}_{3} \mathrm{O}_{4}$ nanoparticles for $\mathrm{Fe}$ (III) ions selective fluorescence sensor. ${ }^{[41]}$ Compared with previous strong acid, organic solvent, complex handling process required research, our proposed sensing method is able to provide an alternative fluorescent nanomaterial of low cost and eco-friendly procedure which can be 
developed as new fluorescent sensing probes for Fe (III) ions detection. Furthermore, it's the first time that FL interactions between the nonpassivated JEFFEMINE ${ }^{\circledR}$ C-dots and Fe (III) ions was studied in details. Our work suggested that the as-prepared C-dots could be successfully applied as a fluorescent probe to detect Fe (III) ions in water rapidly and reliably. 


\section{EXPERIMENT}

\subsection{Carbon Nanodots}

\subsubsection{Reagents}

Ultrapure Millipore water $\left(18.2 \mathrm{M} \Omega, 25^{\circ} \mathrm{C}\right)$ obtained from a Mili Q-system was used throughout the experiments. JEFFEMINE ${ }^{\circledR}$ T3000 and T5000 polyetheramine were provided by HUNTSMAN. Sodium phosphate dibasic ( $\geq 99 \%)$, sodium phosphate monobasic (98\%-102\%), and tetraethyl orthosilicate (99.999\% trace metals basis) were purchased from Sigma-Aldrich (St. Louis, MO). The chloride salt of $\mathrm{Fe}^{3+}$ (97$102 \%$ ACS) was obtained from Alfa Aesar (Ward Hill, MA). The chloride salts of Cu2+ (lab grade), Sr2+ (99+\% ACS), and Zn2+ (99.99\% metals basis) were purchased from Fisher Scientific (Pittsburg, PA). The chloride salts of $\mathrm{Ni2}+(98 \%$ ACS $), \mathrm{Hg} 2+(\mathrm{ACS}>99.5 \%), \mathrm{Na}+(\mathrm{ACS} \geq 99.0 \%), \mathrm{Mn} 2+(99.99 \%$ trace metals basis $)$ and Sn2+ (99.99\% trace metals basis) were purchased from Sigma-Aldrich (St. Louis, MO). All reagents were of analytical grades and used without further purification. Corning plain glass microslides were supplied by Fisher Scientific.

\subsubsection{Synthesis of JEFFEMINE® T3000 and T5000 Carbon Nanodots (C- dots)}

Firstly, $1 \mathrm{~mL}$ JEFFEMINE ${ }^{\circledR}$ T3000 was transferred into a tube with a stir bar and heated in a CEM microwave (Figure 3.1) of $300 \mathrm{~W}$ for 15 cycles, each cycle 
consisted of 1 minute heating and 3 minutes cooling. The CEM with Discover SP and Synergy software ensured the gaseous byproducts from the reaction released to prevent over pressurization and enabled the reacting chamber being temperature and power controlled. The CEM provided precious monitor over the entire synthesis reaction.

The liquid JEFFEMINE ${ }^{\circledR}$ T3000 changed from transparent to dark brown after 15 cycles of heating-cooling for 45 minutes. After cooling down to the room temperature, the homogenous dark brown solution was diluted by $1 \mathrm{~mL}$ ultrapure Ultrapure water and centrifuged for 3 cycles with speed of $7830 \mathrm{rpm}$ in an Eppendorf 5430/5430R centrifuge (Figure 3.2), each cycles took 15 minutes. Finally, a clear golden yellow aqueous solution with well dispersed carbon nanodots was collected. For JEFFEMINE ${ }^{\circledR}$ T5000 C-dots, the completely same method was conducted.

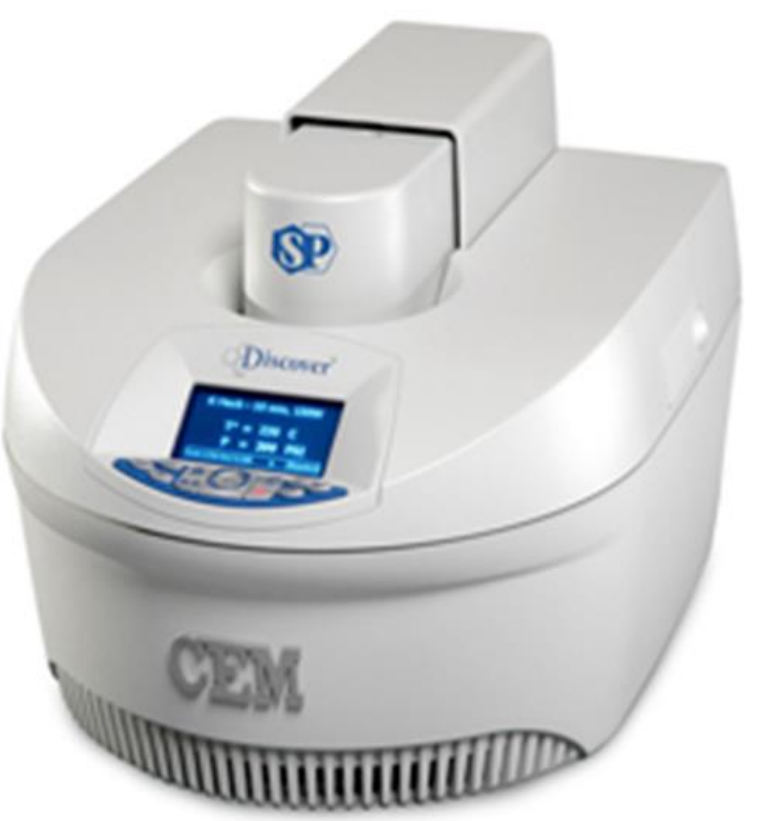


Figure 3.1 Image of CEM microwave used for the synthesis of C-dots

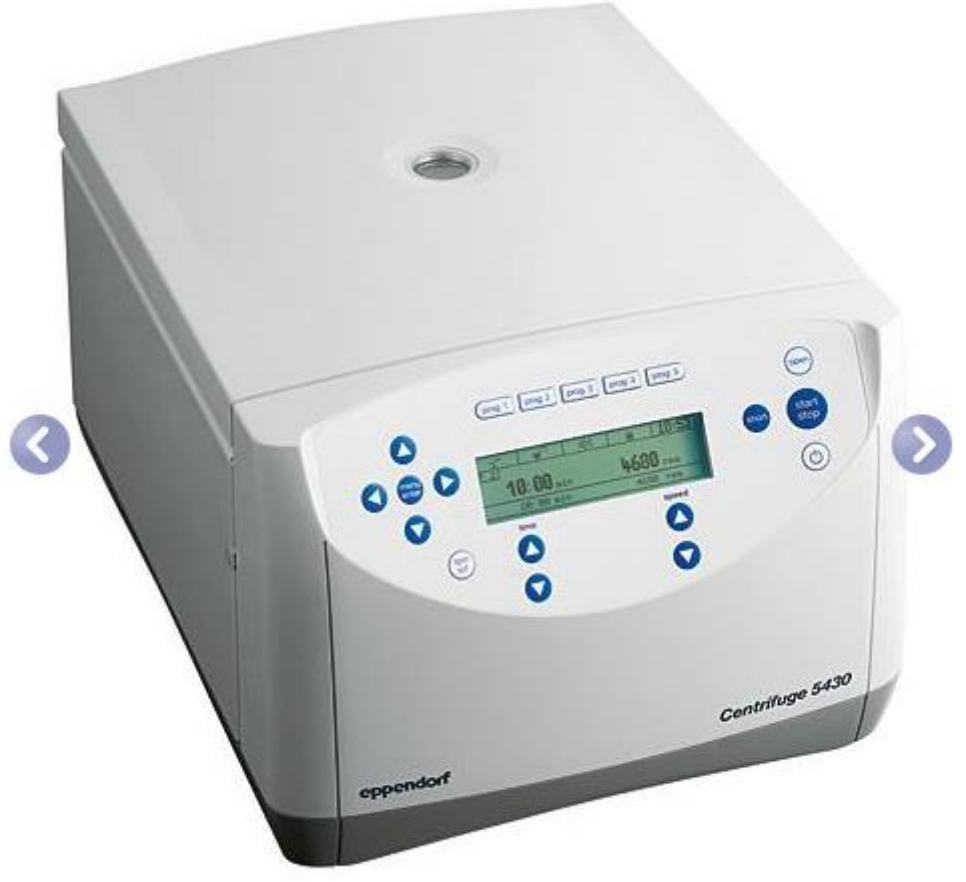

Figure 3.2 Image of effendorf Centrifuge 5430/5430 R

\subsection{Carbon Nanodots immobilized in Sol-Gel Thin Films}

\subsubsection{Sol-Gel Thin Film Preparation}

The sol-gel solution was prepared by dropping a mixture of $0.2 \mathrm{~mL} 1 \mathrm{~N} \mathrm{HCl}$ and 2.8 $\mathrm{mL}$ ultrapure water into $9 \mathrm{~mL}$ of TEOS while stirring in a capped vial with the speed of $1000 \mathrm{rpm}$ at room temperature. The condensation of tons of siloxane bonds was followed by the hydrolysis for 4 hours while stirring at $1500 \mathrm{rpm}$ at room temperature. Immobilized C-dots for sol-gel thin film test in $\mathrm{H}_{2} \mathrm{O}$ was prepared by mixing respectively $0.9 \mathrm{~mL}$ microwave synthesized JEFFEMINE ${ }^{\circledR}$ T3000 and 
T5000 C-dots and $0.1 \mathrm{~mL}$ ultrapure water. $0.1 \mathrm{~mL}$ of diluted C-dots was combined with $0.1 \mathrm{~mL}$ of condensed TEOS solution and the mixture was pipetted onto a glass slide with $1 \mathrm{~cm}$ in width and $2.5 \mathrm{~cm}$ in length, which had been completely cleaned by soaking in $0.1 \mathrm{M} \mathrm{NaOH}$ for at least 10 minutes and rinsing with deionized water before blow dry in nitrogen. Blank thin film sample was prepared by pipetting the mixture of $0.2 \mathrm{~mL}$ ultrapure water and $0.2 \mathrm{~mL}$ condensed TEOS solution on a glass substrate.

A 6800 Spin Coater Series spin coater from Specialty Coating Systems was applied for spin coating the all type of prepared mixture on the glass slides (Figure 3.3).

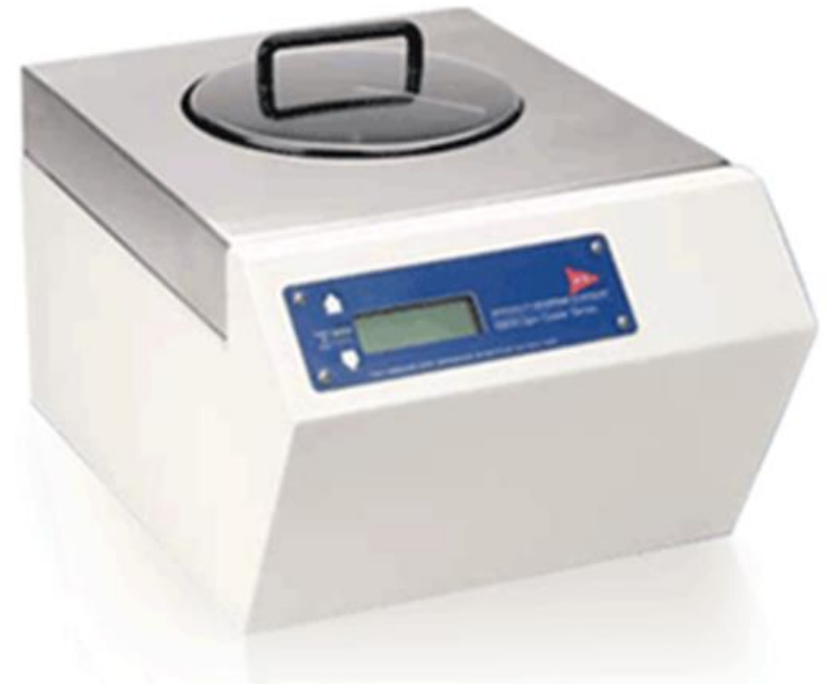

Figure 3.3 6800 Spin Coater Series spin coater from Specialty Coating Systems 


\subsection{Characterization}

\subsubsection{TEM}

The morphology of C-dots was studied by high resolution transmission electron microscope (TEM). A Tecnai G² F30 Twin from FEI Company equipped with a 300 $\mathrm{kV}$ field emission gun was applied for TEM characterization which provided higher resolution (Figure 3.4). Holy copper grids were dipped into the oven treated brown concentrated T3000 and T5000 C-dots followed by being washed with aceton and subsequently air-dried for 5 minutes at room temperature.

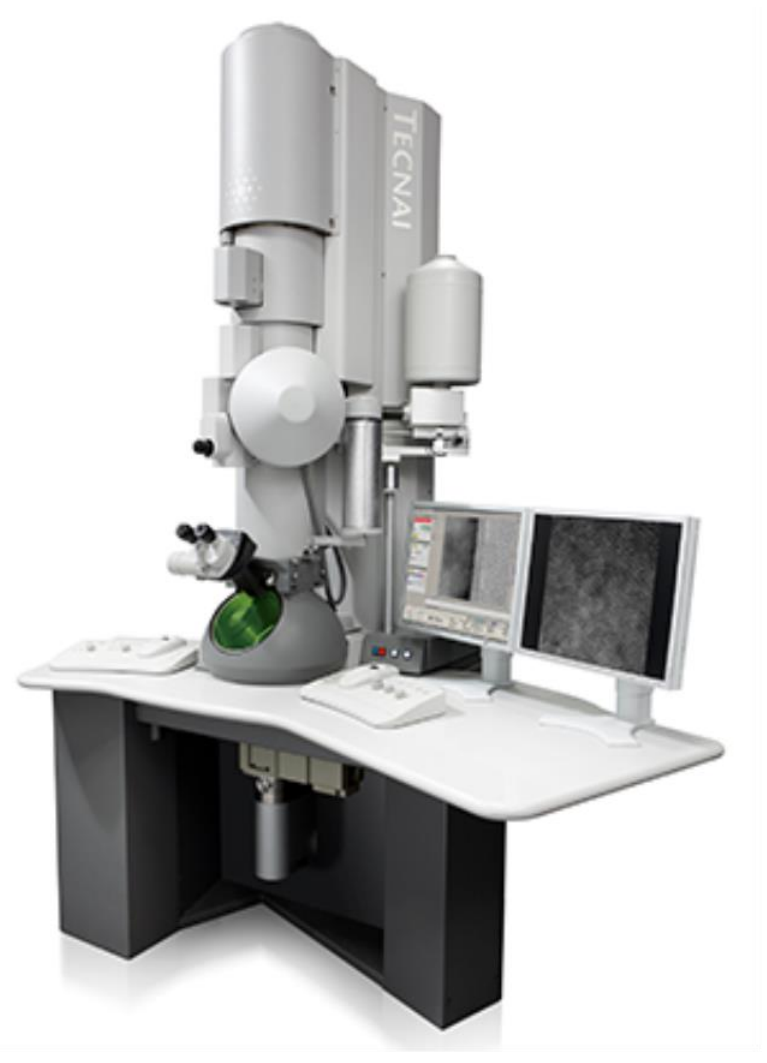

Figure 3.4 Tecnai $\mathrm{G}^{2} \mathrm{~F} 30$ Twin from FEl Company equipped with a $300 \mathrm{kV}$ field emission gun 


\subsubsection{AFM}

Atomic force microscope (AFM) images were obtained on 5500 AFM from Agilent Technologies. AFM C-dots sample was prepared by concentrating $1 \mathrm{~mL}$ T3000 and T5000 C-dots solution separately in two clean glass vials set in the furnace oven at $50{ }^{\circ} \mathrm{C}$ for 3 hours after centrifugation had been done. The golden transparent liquid C-dots solution changed to brown viscous one after 3 hours heating. $2 \mu \mathrm{L}$ concentrated $\mathrm{C}$-dots were diluted with $40 \mu \mathrm{L}$ ultrapure water and total $5 \mu \mathrm{L}$ diluted C-dots were pipetted on a piece of mica. The AFM scanning was performed 20 minutes after the sample on the mica piece was completely air dried at room temperature. The scanning area was $1500 \times 1500 \mathrm{~nm}$.

\subsubsection{FTIR}

Fourier transform infrared spectroscopy (FTIR) spectra of the samples was measured on a Nicolet 4700 equipped with a Thermo Smart Performer germanium crystal ATR attachment from Thermo Scientific. The resolution performed on Cdots sample was $1 \mathrm{~cm}^{-1} .70 \mu \mathrm{L}$ microwave synthesized T3000 C-dots were mixed with $30 \mu \mathrm{L}$ condensed TEOS in a glass vial and pipetted on a piece of silica slide (1 $\mathrm{cm}$ in length, $1 \mathrm{~cm}$ in width). SCS 6800 spin coater was used for FTIR silica thin film. The speed was $1000 \mathrm{rpm}$ and dwell time was $30 \mathrm{~s}$. The same procedure was followed for the preparation of T5000 C-dots FTIR silica thin film.

\subsubsection{Absorbance}

UV-Vis spectra absorbance was recorded on a BioTek Powerwave HT Microplate Spectrophotometer recording spectra with $2 \mathrm{~nm}$ interval and at medium scan rate. 
No interference filter is required, and this plate reader can provide continuous wavelength selection from $200 \mathrm{~nm}$ to $999 \mathrm{~nm}$.

All C-dots were diluted with ultrapure water in quartz cuvettes with a $1 \mathrm{~cm}$ path length. Blank correction was performed on all spectra.

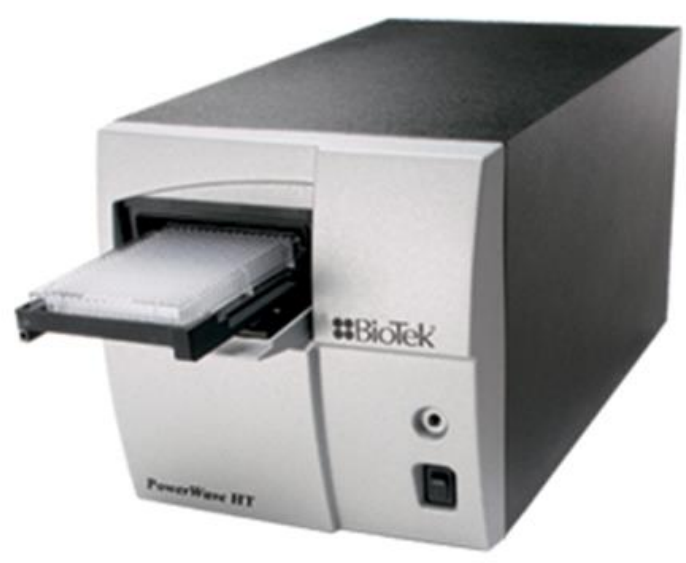

Figure 3.5 Image of BioTek Powerwave HT Microplate Spectrophotometer, used for the detection UV-Vis absorption spectra of C-dots

\subsubsection{Fluorescence}

Fluorescence intensity of JEFFAMINE ${ }^{\circledR}$ T3000 and T5000 C-dots was analyzed on a Horiba Fluorolog spectrofluorometer (Figure 3.5) with slit width of $2 \mathrm{~nm} .0 .1$ $\mathrm{mL}$ T3000 and T5000 C-dots were diluted with $2.5 \mathrm{~mL}$ ultrapure water separately in quartz cuvettes with $1 \mathrm{~cm}$ path length. The T3000 C-dots were excited at 375 $\mathrm{nm}$ with a strong emission peak obtained at $448 \mathrm{~nm}$, and the excitation wavelength of the T5000 C-dots was $365 \mathrm{~nm}$ with maximum emission at $439 \mathrm{~nm}$. Blank background correction was applied with $2.5 \mathrm{~mL}$ ultrapure water in the same quartz cuvettes excited with the same wavelength as the one did on diluted C-dots. 
Excitation wavelength dependent emission shift was also studied on the prepared C-dots with slit width of $2 \mathrm{~nm}$. The emission wavelength started from $325 \mathrm{~nm}$ to $405 \mathrm{~nm}$ with $10 \mathrm{~nm}$ increments.

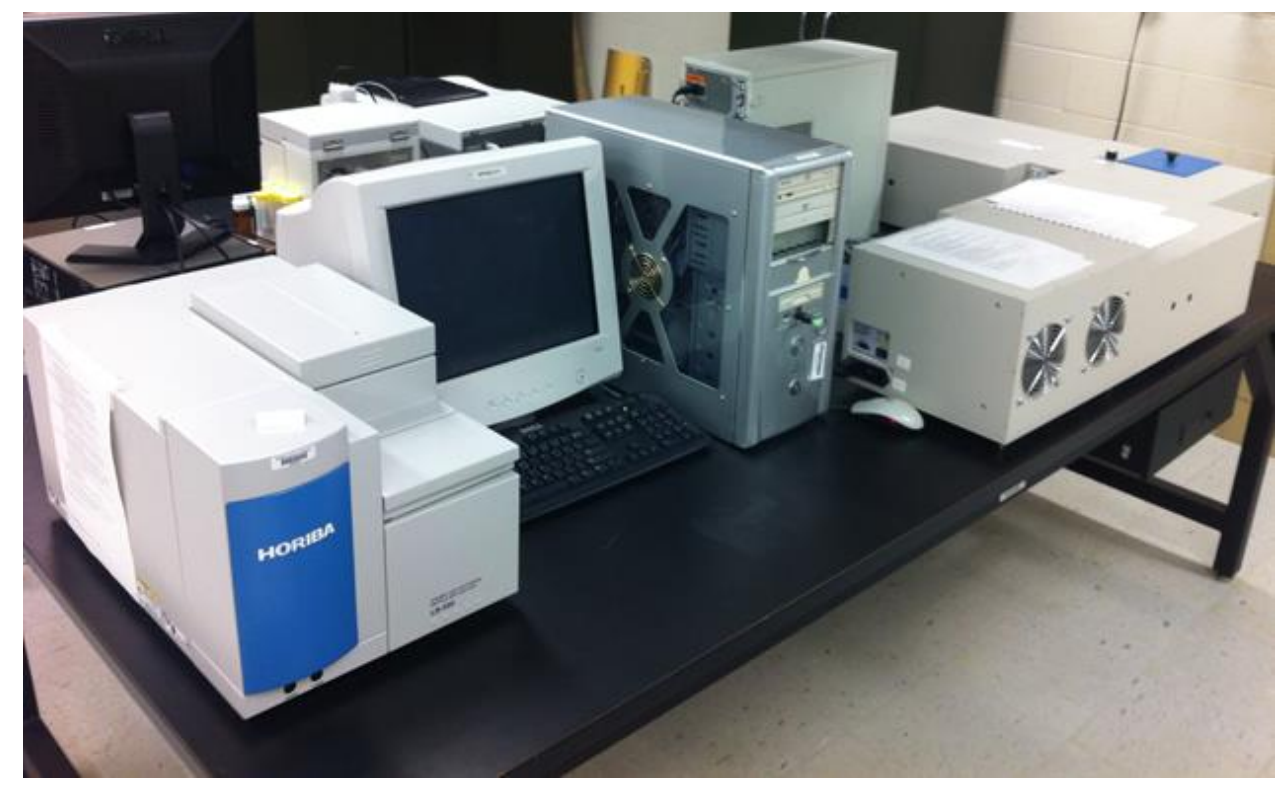

Figure 3.6 Horiba Fluorolog Spectrafluorometer

\subsection{Metal ions detection}

\subsubsection{Metal ions detection in $\mathrm{H} 2 \mathrm{O}$}

Metal ions detection was performed both in ultrapure water and $25 \mathrm{mM}$ phosphate buffer ( $\mathrm{pH}$ 7.4). For the test in $\mathrm{H}_{2} \mathrm{O}, 0.1 \mathrm{~mL}$ of $\mathrm{T} 3000$ and $\mathrm{T} 5000$ was diluted in 2.5 $\mathrm{mL}$ of ultrapure water respectively. As for the test performed in buffer solution, the two kind of C-dots were diluted in $2.5 \mathrm{~mL}$ of $25 \mathrm{mM}$ phosphate buffer. To explore the selectivity of T3000 and T5000 C-dots towards Fe (III) ions over other ions in water and buffer, the emission spectra were collected at ion concentrations of 0 , 
$500,1000,1500,2000,2500,3000,3500$, and $4000 \mu \mathrm{M}$ using $375 \mathrm{~nm}$ and 365 nm excitation for T3000 and T5000 C-dots respectively. The excitation and emission slits used were $2 \mathrm{~nm}$. For the sensitivity and linear respond range of the two type of C-dots upon the Fe (III) ions in water, the emission spectra were collected at ion concentrations of $0,5,25,50,75,100,250$ and $500 \mu \mathrm{M}$. All result was background corrected using $\mathrm{H}_{2} \mathrm{O}$.

\subsubsection{Metal ions detection in sol-gel thin films}

Metal ion sensing in sol-gel thin film was conducted by setting the glass substrates spin coated with sol-gel entrapped C-dots in quartz cuvettes containing $2.5 \mathrm{~mL}$ ultrapure water. Emission spectra were measured at ions concentrations of 0,500 , $1000,1500,2000,2500,3000,3500$, and $4000 \mu \mathrm{M}$ using $375 \mathrm{~nm}$ and $365 \mathrm{~nm}$ excitation wavelength. Each measurement was performed 15 minutes after the ions solution added. All result was background corrected using $\mathrm{H}_{2} \mathrm{O}$. 


\section{RESULT and DISCUSSION}

\subsection{Carbon Nanodots}

\subsubsection{Synthesis of Carbon Nanodots}

Carbon nanodots were synthesized from JEFFAMINE ${ }^{\circledR}$ T3000 and T5000 (Figure 4.1) as carbon source and with no passivation reagent. The transparent organic compound changed to dark brown solution, followed by 15 heating-cooling cycles in a $300 \mathrm{~W}$ microwave for 45 minutes. $1 \mathrm{~mL}$ ultrapure water was added to the cool down dark brown solution before centrifugation to extract C-dots from the solution. Finally, C-dots particles were well dispersed in the golden-yellow colored aqueous supernatant. The possible mechanism of C-dots formation may include four steps: dehydration, polymerization, pyrolysis and self-passivation. ${ }^{[1]}$
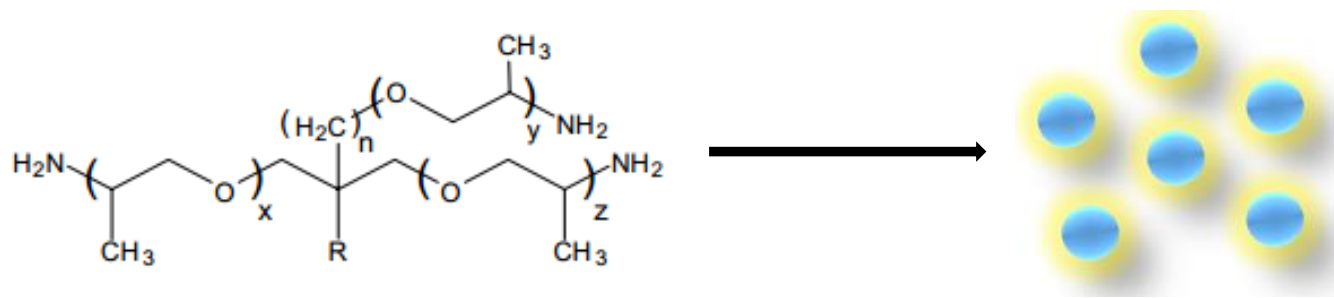

Figure 4.1 Depiction of microwave-assisted synthesis of carbon nanodots from JEFFAMINE ${ }^{\oplus}$ T series which is examplified by the above structure, for JEFFAMINE ${ }^{\circledR}$ T3000, $R=H, n=0, x+y+z=50, M W=3000$; for JEFFAMINE ${ }^{\circledR}$ T5000, $R=H, n=0, x+y+z=50$, $\mathrm{MW}=5000$. 


\subsection{Carbon Nanodots Sol-Gel Thin Films}

\subsubsection{Sol-Gel Thin Film Preparation}

The sol-gel solution was prepared by dropping a mixture of $0.2 \mathrm{~mL} 1 \mathrm{~N} \mathrm{HCl}$ and $2.8 \mathrm{~mL}$ ultrapure water into $9 \mathrm{~mL}$ of TEOS while stirring in a capped vial with the speed of $1000 \mathrm{rpm}$ at the room temperature. C-dots sample for sol-gel thin-film testing was prepared by mixing $0.9 \mathrm{~mL}$ microwave synthesized JEFFEMINE ${ }^{\circledR}$ T3000 or T5000 C-dots and $0.1 \mathrm{~mL}$ ultrapure water. $0.1 \mathrm{~mL}$ of diluted C-dots sample was combined with $0.1 \mathrm{~mL}$ of condensed TEOS solution and the mixture was pipetted onto a glass slide with $1 \mathrm{~cm}$ in width and $2.5 \mathrm{~cm}$ in length.

\subsection{Characterization}

\subsubsection{TEM}

Transmission electron microscope (TEM) images of as-prepared JEFFAMINE ${ }^{\circledR}$ T3000 and T5000 carbon nanodots (Figure 4.2) were obtained and demonstrated that C-dots were exhibited as uniform, spherical particles mono-dispersing in the water. Also, some lattice fringes can be observed in TEM images (Figure 4.2c, 4.2d) revealing the detailed structurally crystalline morphology of C-dots. The size distribution chart (Figure 4.2e, 4.2f) indicated that the mean size was estimated to be $4.27 \pm 0.79 \mathrm{~nm}$ and $3.81 \pm 0.80 \mathrm{~nm}$ for the T3000 and T5000 C-dots, respectively. 
a

\section{0. $\mathrm{nm}$}

b

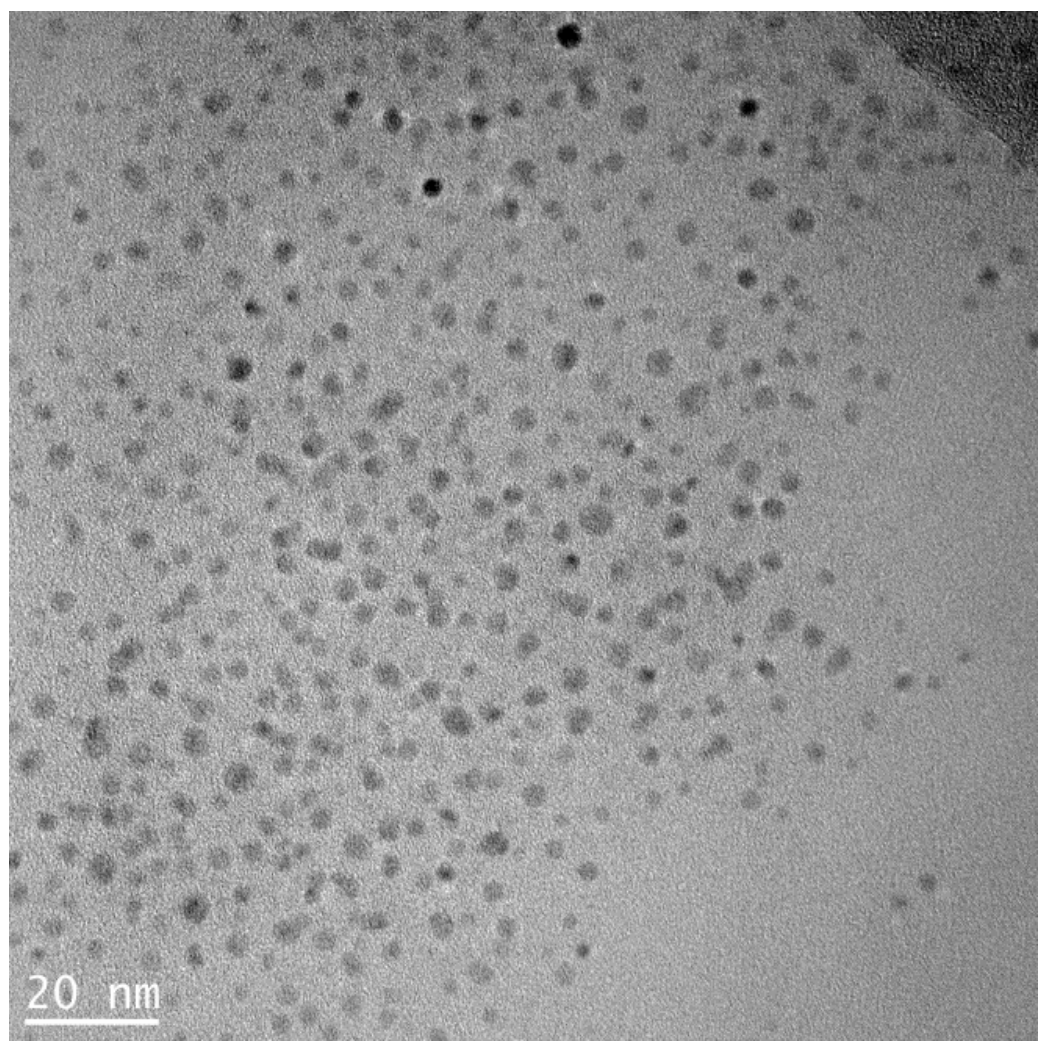



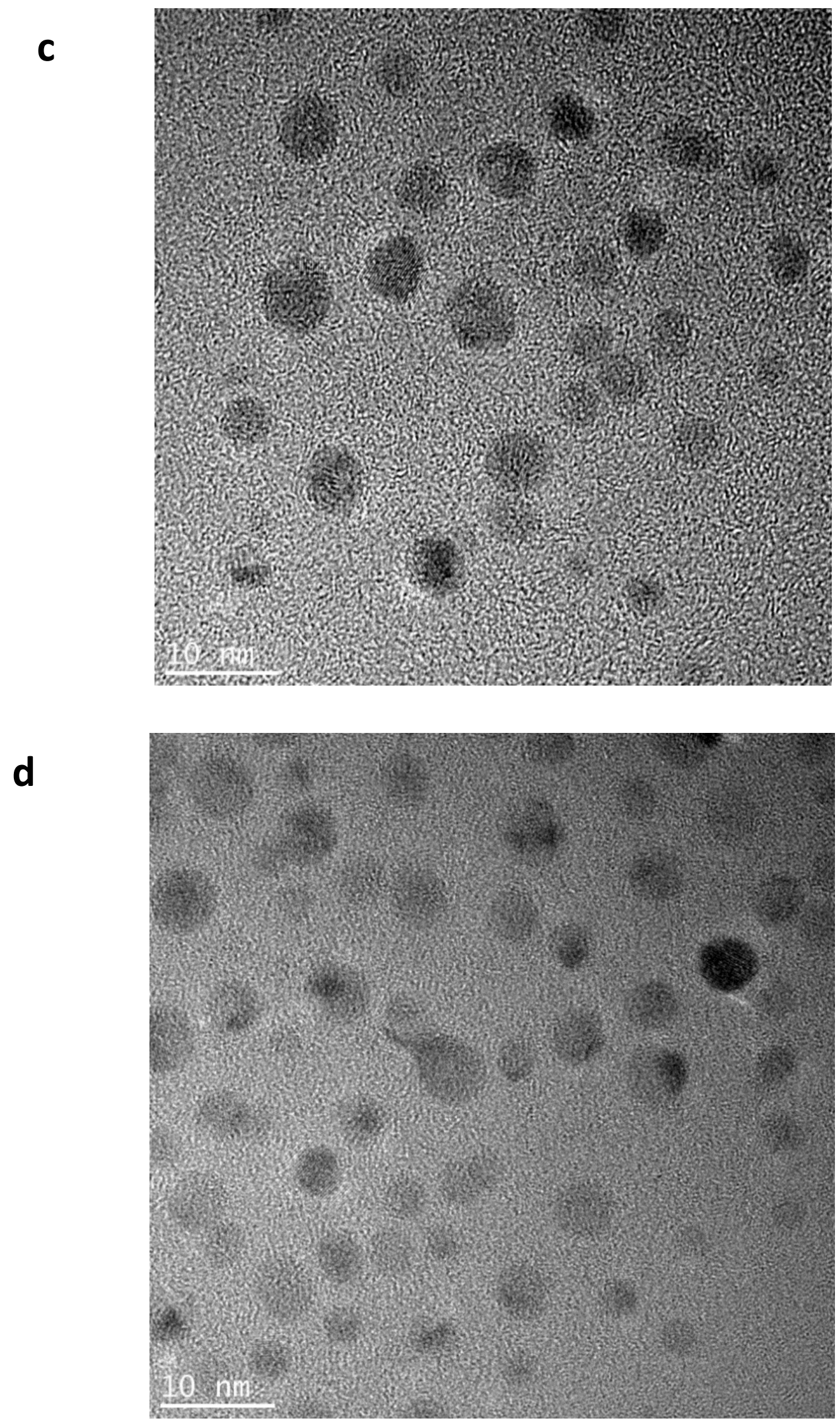
e
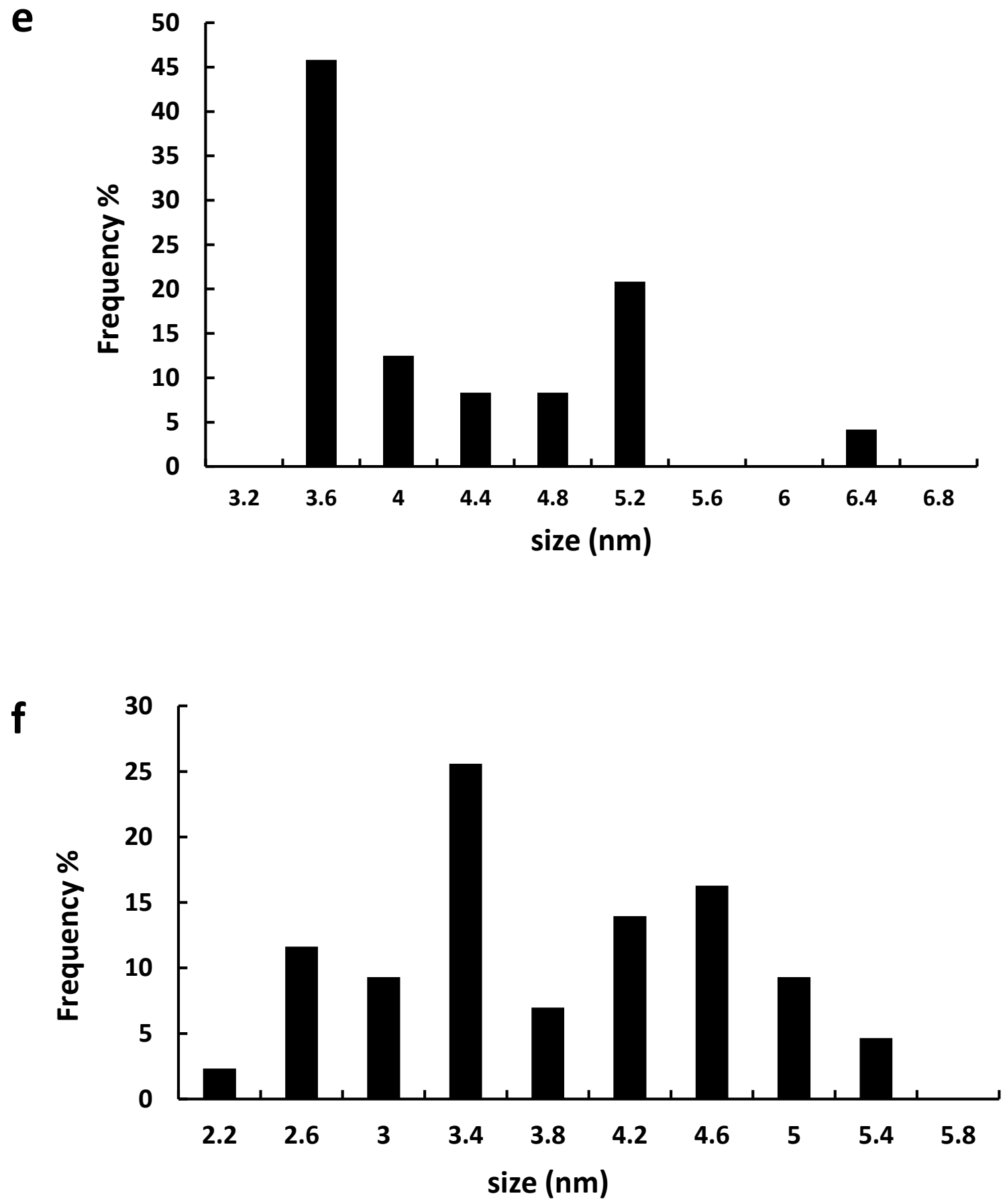

Figure 4.2 TEM image of C-dots (a),(c) T3000 C-dots (b),(d) T5000 C-dots, scale bar: (a),(b) $20 \mathrm{~nm}$; (c) (d) $10 \mathrm{~nm}$; and the corresponding particle size distribution of as prepared (e) T3000 C-dots, (f) T5000 C-dots. 


\subsubsection{AFM}

Atomic force microscopy (AFM) allowed the acquisition of images of both C-dots sample (Figure 4.3), indicating that uniform C-dots particles were mono-dispersing on the mica which was well consistent with the TEM result. From the crosssectional height analysis (Figure 4.3e, 4.3f), the average thickness of these two group of representative C-dots were nearly $1 \mathrm{~nm}$. As shown on Fig $2 \mathrm{e}$ and $2 \mathrm{f}$, the mean height of T3000 C-dots were $0.949 \mathrm{~nm}$, around $0.2 \mathrm{~nm}$ higher than that of T5000 C-dots. Thus it's reasonable to believe that C-dots produced from JEFFAMINE ${ }^{\circledR}$ T5000 had finer dimensions than those made from JEFFAMINE ${ }^{\circledR}$ T3000, which may be a possible source that led to their different interference ability to metal ions.

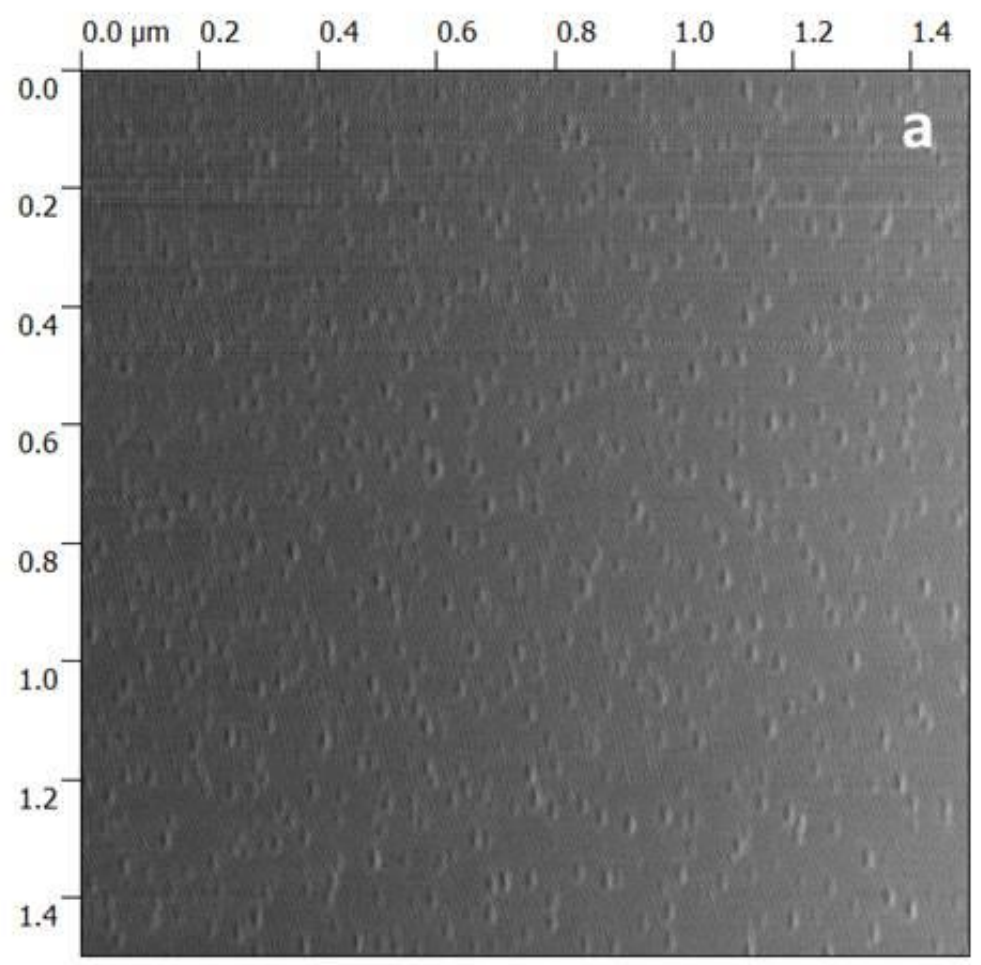



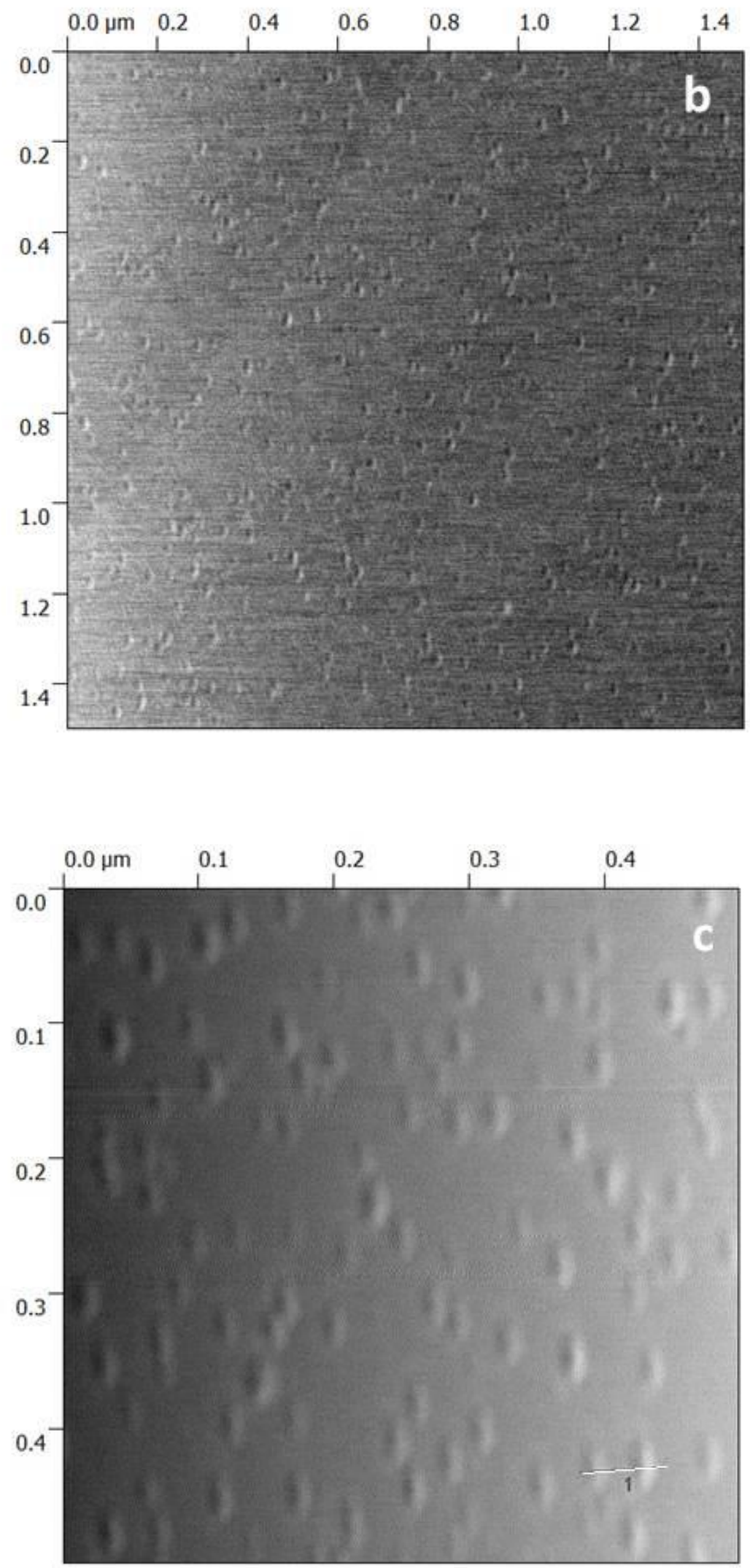

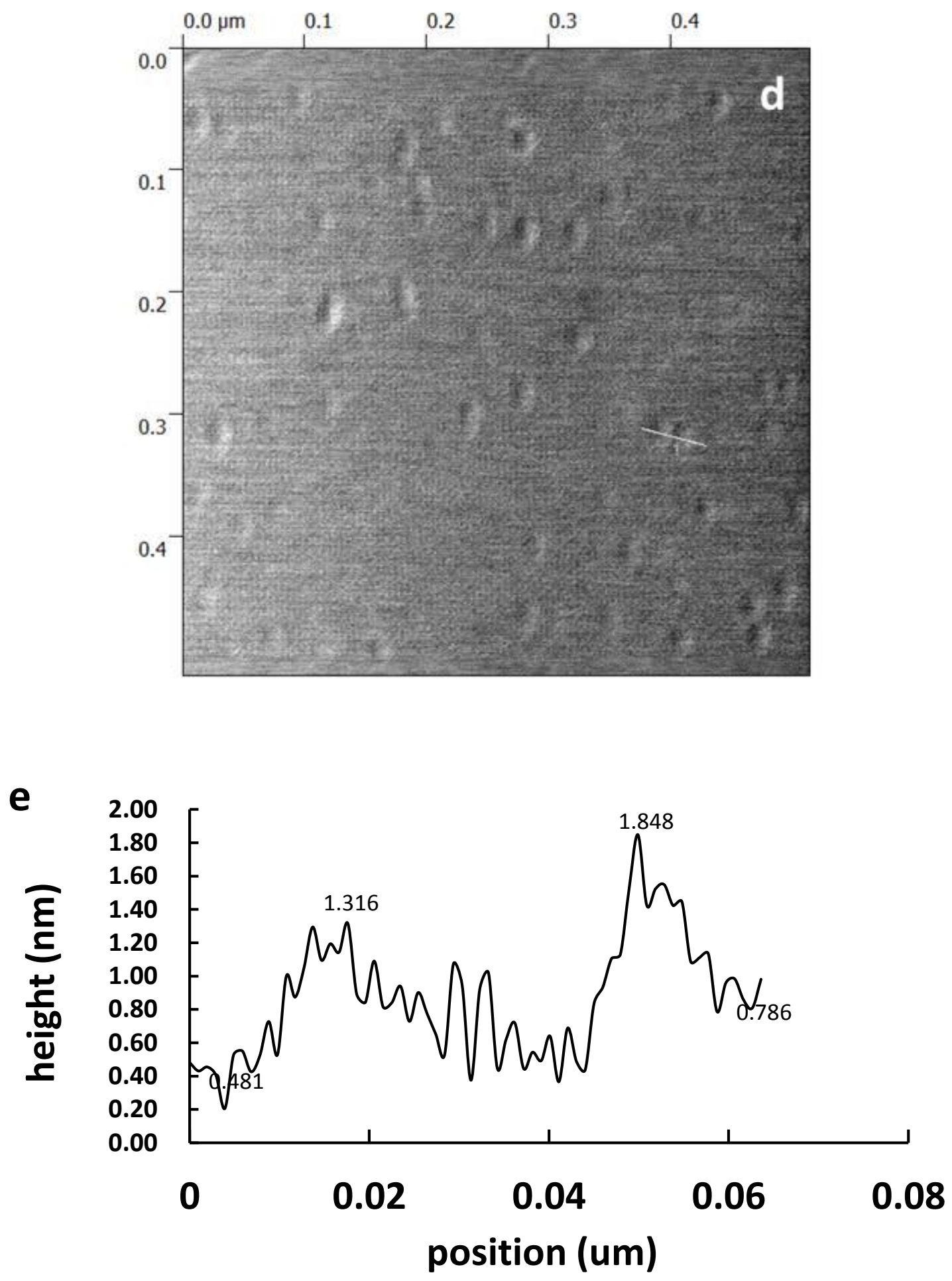


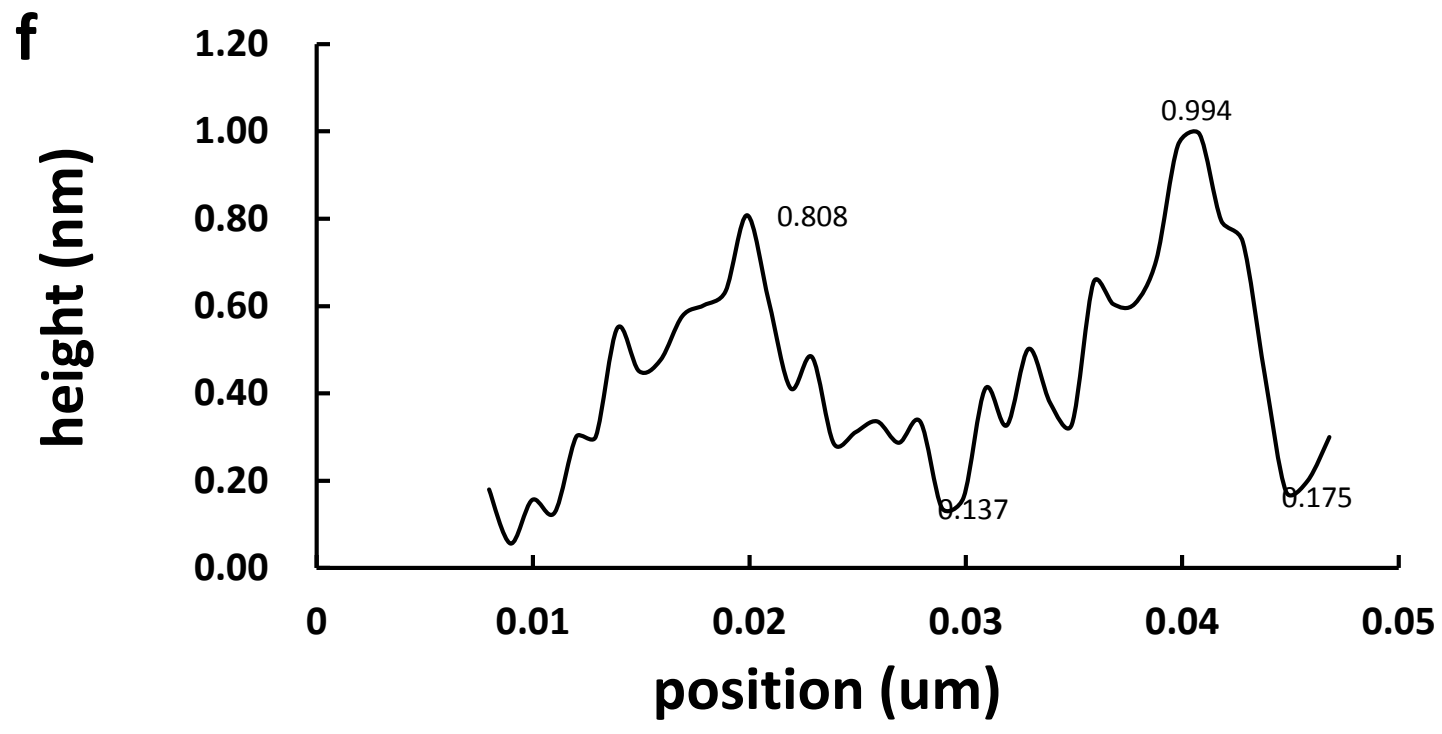

Figure 4.3 AFM images for T3000 and T5000 C-dots with different magnification and the height analysis of representative (a)(c)(e) T3000 C-dots; (b)(d)(f) T5000 C-dots both dispersed in water

\subsubsection{FTIR}

To study the functional groups on the surface of as-prepared C-dots, Fourier Transform infrared spectra (FTIR) were applied for characterization. Two samples showed almost the same characteristic in their spectra (Figure 4.4). Compared to pure TEOS (sample background), the vibration of $\mathrm{C}-\mathrm{OH}$ bonds $\left(\sim 3350 \mathrm{~cm}^{-1}\right)$ detected on two samples were weak but measureable, which may be resulted from the amorphous structure inside the C-dots core. Bands at $2750-3000 \mathrm{~cm}^{-1}$ determined the stretching vibration of $\mathrm{C}-\mathrm{H}$ groups. The result attributed to the possible existence of hydroxyl groups on the surface of T3000 and T5000 C-dots. 

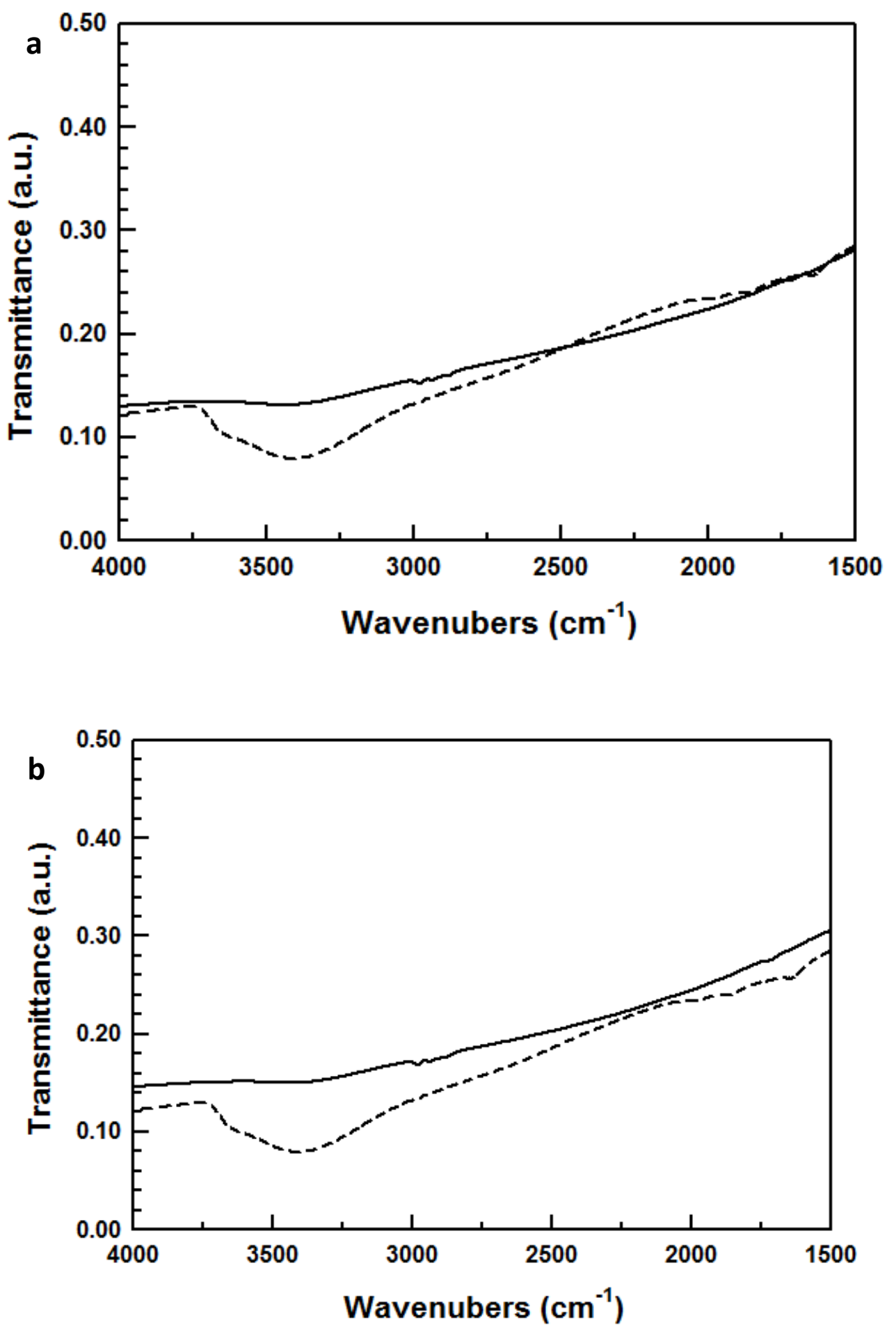

Figure 4.4 FTIR spectra of (a) T3000 C-dots (solid line) and TEOS, (b) T5000 C-dots (solid line) and TEOS 


\subsubsection{Fluorescence}

C-dots prepared from JEFFAMINE ${ }^{\circledR}$ T3000 and T5000 exhibited strong PL with no additional surface passivation step. The UV-Vis spectra showed each sample had two small absorbance bands centered at 292/360 nm 293/360 nm respectively (Figure 4.5). The band near 290 was attributed to the possibility of the $n \rightarrow \pi^{*}$ transition of $\mathrm{C}=\mathrm{O}$ on the surface of the $\mathrm{C}$-dots. Correspondingly, the strongest $\mathrm{FL}$ was obtained at $448 \mathrm{~nm}$ when T3000 C-dots were excited at $375 \mathrm{~nm}$, and the maximum intensity of T5000 C-dots at $439 \mathrm{~nm}$ was observed at the $365 \mathrm{~nm}$ excitation wavelength. Bright green-blue light was emitted by the two samples under UV lamp excitation which was visible for naked eye ${ }^{[42]}$. Based on the previous published literature, it was suggested that the origin emission of the Cdots are partly size dependent, which means that the better photoluminescence efficiency will be obtained with smaller size of C-dots. ${ }^{[43]}$ Our C-dots prepared via the microwave pyrolysis methods were both less than $5 \mathrm{~nm}$ in diameter, which may contribute to the observed strong visible photoluminescence. 
a
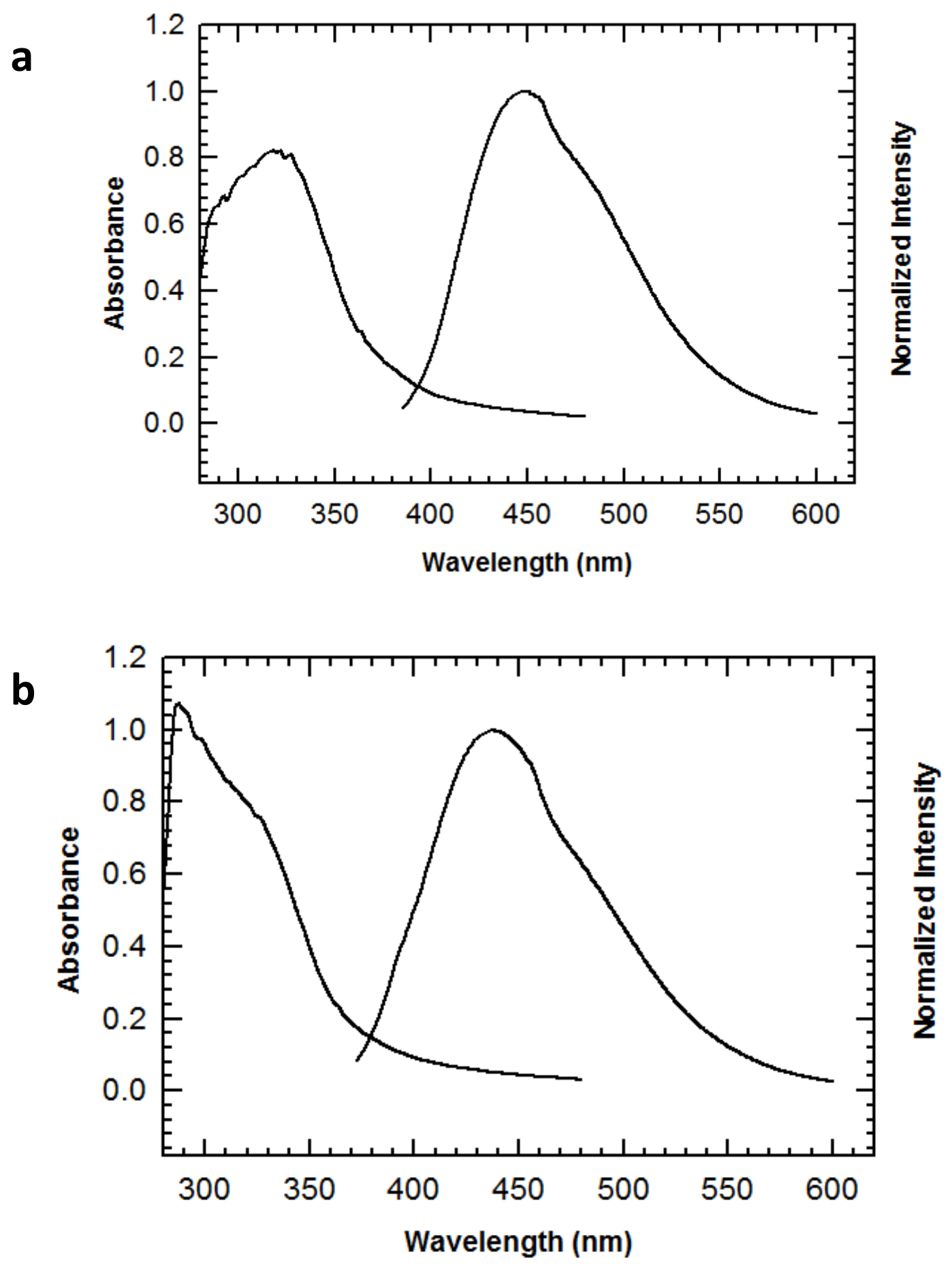

Figure 4.5 UV absorbance spectra and normalized emission spectra of C-dots prepared from JEFFAMINE $\circledast$ (a) T3000 $\left(\lambda_{\mathrm{ex}}=\mathbf{3 7 5} \mathrm{nm}\right)$ and $(\mathrm{b})$ T5000 $\left(\boldsymbol{\lambda}_{\mathrm{ex}}=\mathbf{3 6 5} \mathbf{n m}\right)$. 
JEFFAMINE ${ }^{\circledR}$ C-dots also showed the feature of the $\lambda_{\text {ex }}$ dependent emission (Figure 4.6). The resulting photoluminescence spectra of JEFFAMINE ${ }^{\circledR}$ C-dots were spectrally broad in visible spectrum, with peak intensities wavelength ranging from $400 \mathrm{~nm}$ to $500 \mathrm{~nm}$ depending on the excitation wavelengths from 325 to 405 nm. As shown in Figure 4.6, the emission peak position of the two C-dots red shifted to longer wavelength with the increasing excited wavelength. Although the PL mechanism of carbon nanodots has not been entirely known and requires further investigation, there are studies showed that functional groups of precursor can be charged responsibility for one of the reasons that can explain the origins of the PL emitted from C-dots. A combination of amino and carboxyl groups in the JEFFAMINE ${ }^{\circledR}$ compound may enhance the most fluorescence activity of our Cdots. ${ }^{[2,44]}$ In addition, it is considerable possible that the different size and surface traps for emission of each carbon nanodots that also attributed the variation emission condition of these microwave synthesized C-dots. 

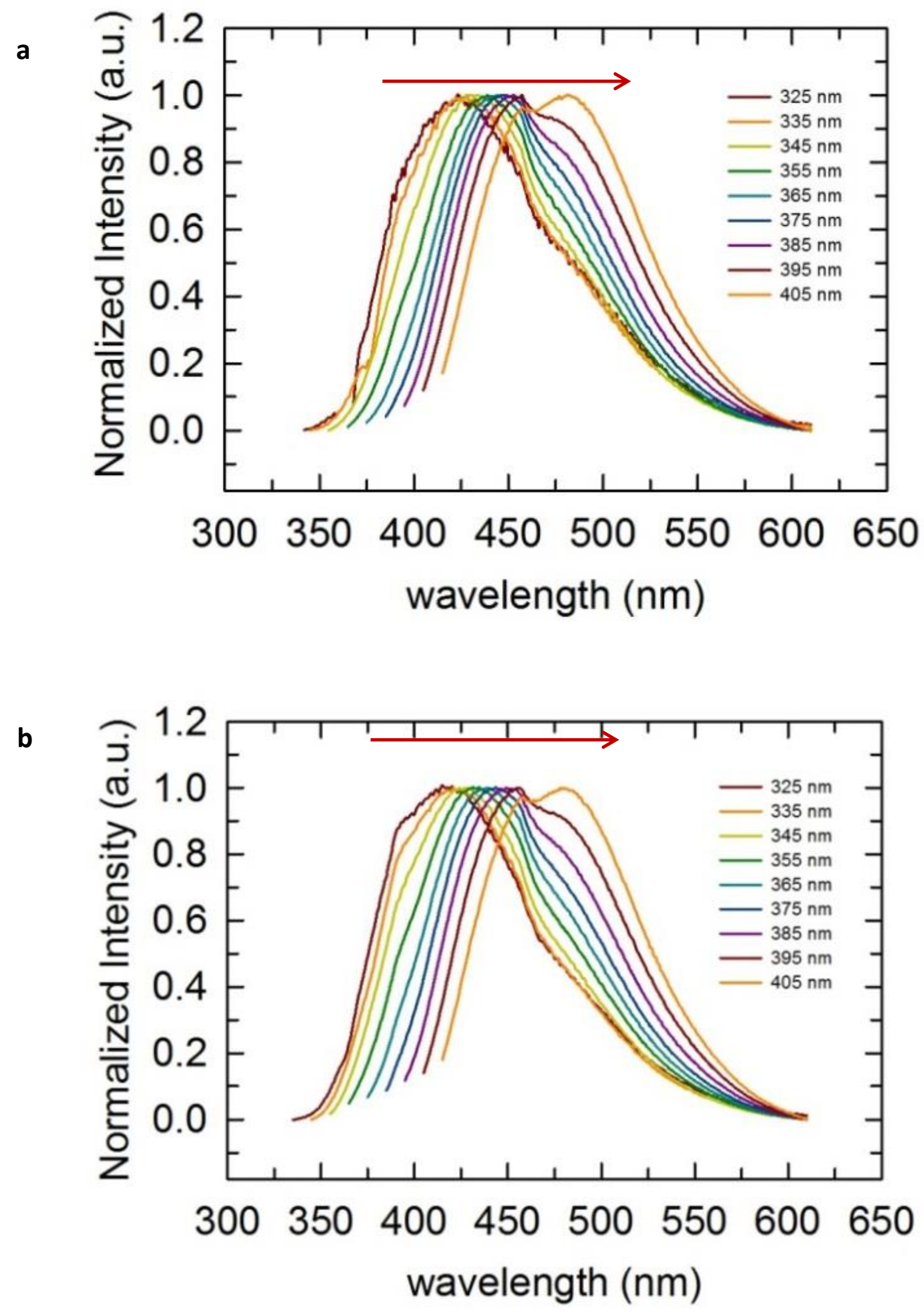
Figure 4.6 Normalized fluorescence emission spectra with increasing longer excitation wave length (in $10 \mathrm{~nm}$ increments starting from $325 \mathrm{~nm}$ ) of C-dots in Ultrapure water produced from microwave irradiated a) JEFFAMINE® T3000; b) JEFFAMINE® T5000.

\subsection{Metal Ion Sensing}

\subsubsection{Ion Sensing in Solution}

Fluorescence quenching has demonstrated to be an effective analytical approach employed to trace and quantify various metal ions for environmental and health concern. Although intensive effort has been devoted into the studied for the sensing application of semiconductor quantum dots, which present ideal physicalchemical properties as a stable kind of optical sensor, the fact of possible heavy metal leaching and coat intracellular/in vivo degradation significantly increase the risk to human body and environment. As a result, the selectivity of this JEFFAMINE $^{\circledR}$ T3000 and T5000 C-dots, alternative nano-scale sensors, were investigated by monitoring the fluorescence intensity of both type of C-dots in the presence of 9 different heavy metal ions, including $\mathrm{Sn}(\mathrm{II}), \mathrm{Hg}(\mathrm{II}), \mathrm{Cu}(\mathrm{II}), \mathrm{Na}(\mathrm{I})$, $\mathrm{Ni}(\mathrm{II}), \mathrm{Zn}(\mathrm{II}), \mathrm{Mn}(\mathrm{II}), \mathrm{Fe}(\mathrm{III}), \mathrm{Sr}(\mathrm{II})$, which are either cellular cations or exist in

organisms. ${ }^{[32]}$ And this fluorescence intensity response detection was carried in water. In order to correct the possible inner filter effect from the yellow-colored Fe (III) ions solution, only points below $500 \mu \mathrm{M}$ were analyzed in our sensing research. Remarkably, as indicated in Figure 4.7, T3000 C-dots showed the strongest sensing ability for detecting Fe (III) ions in water. The FL intensity of the T3000 C- 
dots were showed largely quenched upon the addition of Fe (III) ions in water (Figure 4.8). T5000 C-dots showed slightly poorer sensing ability towards the Fe (III) ions compared with the T3000 C-dots did. The significant higher relative FL intensity ( $\mathrm{F}_{0} / \mathrm{F}$ ratio) of both T3000 and T5000 C-dots sample upon the increasing addition of Fe (III) ions in water suggested the prime sensitivity of this sensing system, leading to their reliable and visible detecting capability and excellent potential for the applications in various areas especially biological sensing in the near future.

The complexity of environment and biological system highly requires the analytical methods for heavy metal ions detection not only in sensitivity but more importantly in selectivity. ${ }^{[32]}$ The performed selectivity experiment result that tested metal ions, besides Fe (III), barely deactivated the fluorescence intensity of neither T3000 Cdots nor T5000 sample in water. This metal ions control test supported the fact that due to their excellent sensitivity and selectivity profile, the new JEFFAMINE ${ }^{\circledR}$ T3000 and T5000 C-dots will be a prospective potential candidate for metal ion sensing in water. 


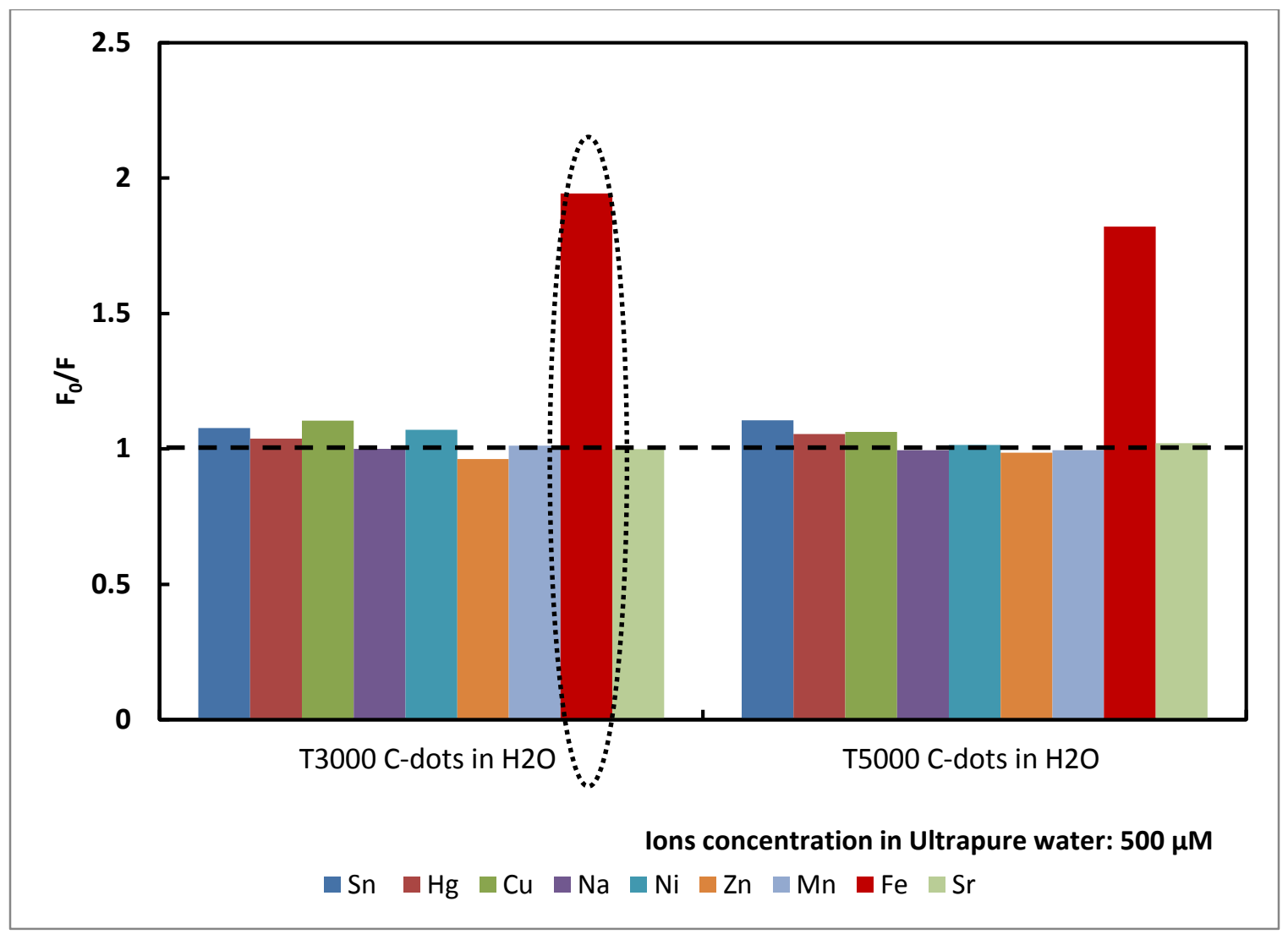

Figure 4.7 Fluorescence response of T3000 and T5000 C-dots towards various metal ions, where each sample $(0.1 \mathrm{ml})$ was diluted in deionized water $(2.5 \mathrm{ml})$. The concentration of metal ions was $500 \mu \mathrm{M}$. Where $\mathrm{F}_{0}$ and $\mathrm{F}$ represents respectively the $\mathrm{FL}$ intensities of C-dots in the absence and presence of $500 \mu \mathrm{M}$ metal ions in $\mathrm{H}_{2} \mathrm{O}$. Dash circle presented the T3000 C-dots diluted with water to be the most sensitive and selective sensing system (illustrated in Figure 4.8). 


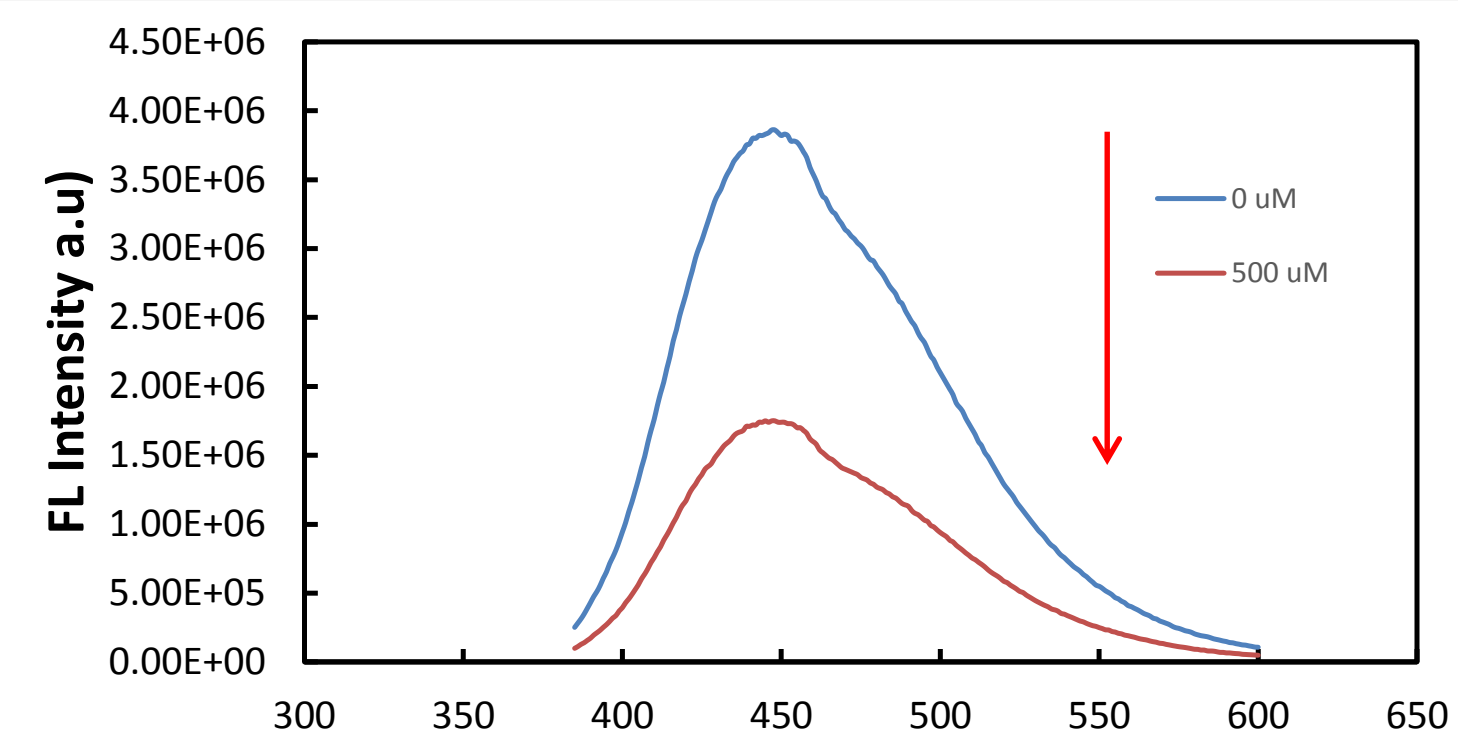

Wavelength (nm)

Figure 4.8 Fluorescence response of T5000 C-dots towards the addition of Fe (III) ions in water (concentration of $\mathrm{Fe}(\mathrm{III}): 0,500 \mu \mathrm{M}$ )

To further evaluate the analytical performance of T3000 and T5000 C-dots working as sensitive and selective sensors to qualify and quantify the $\mathrm{Fe}$ (III) ions in $\mathrm{H}_{2} \mathrm{O}$, solution containing different concentrations of Fe (III) ions was added in to the diluted C-dots solution and the fluorescent spectra of T3000 and T5000 C-dots were recorded respectively. Figure 4.9 revealed that the emission intensity of both T3000 and T5000 C-dots were quenched by Fe (III) gradually in water with the concentrations of $\mathrm{Fe}$ (III) ions increasing from 0 to $500 \mu \mathrm{M}$. Stern-Volmer equation is one of the reliable equations that can describe the concentration dependent chemo-sensor as reported in various literatures. ${ }^{[33]}$

$$
\mathrm{F}_{0} / \mathrm{F}=1+\mathrm{K}_{\mathrm{sv}} \mathrm{CFe}_{\mathrm{Fe}}
$$


$\mathrm{F}_{0}$ and $\mathrm{F}$ are respectively the fluorescence intensities in the absence and presence of $\mathrm{Fe}$ (III) ions, an effective quencher as studied. $\mathrm{C}_{\mathrm{Fe}}$ presents the $\mathrm{Fe}$ (III) ions concentration, and $\mathrm{K}_{\mathrm{sv}}$ is the quenching constant. A Stern-Volmer plot with a linear relationship between the relative fluorescence intensity $\left(F_{0} / F\right)$ and the concentration of Fe (III) ions was illustrated in Figure 4.9c, 4.9d. Correlation coefficients were 0.9985 and 0.9998 with quenching constant $K_{\mathrm{sv}}$ of $2.4 \times 10^{3}$ and $2.7 \times 10^{3} \mathrm{M}^{-1}$ respectively for T3000 and T5000 C-dot, which indicated that both type of microwave synthesized C-dots can be sensitive detector for Fe (III) ions in water (Table 4.1). Although the reasons for this quenching mechanism with high selectivity and sensitivity are not totally clear at the present stage, one of the possible explanations might be the good coordination of Fe (III) ions, that allowed the formation of complex network between the iron ions and oxygen-containing or nitrogen-containing groups on the surface of the C-dots. ${ }^{[45]}$ The interference of the complex formation could be one of the disturbances to the energy emission of the fluorescence probe. ${ }^{[29]}$ Meanwhile, Fe (III) ions have been reported higher oxidation potential for $\mathrm{Fe}^{3+} \rightarrow \mathrm{Fe}^{2+}$ which means $\mathrm{Fe}$ (III) ions are considerably good electrons acceptor promoting electron transfer during the fluorescence quenching process. As a result, Fe (III) ions had much stronger interference over other tested cations on the FL properties of the T3000 and T5000 C-dots sample. 

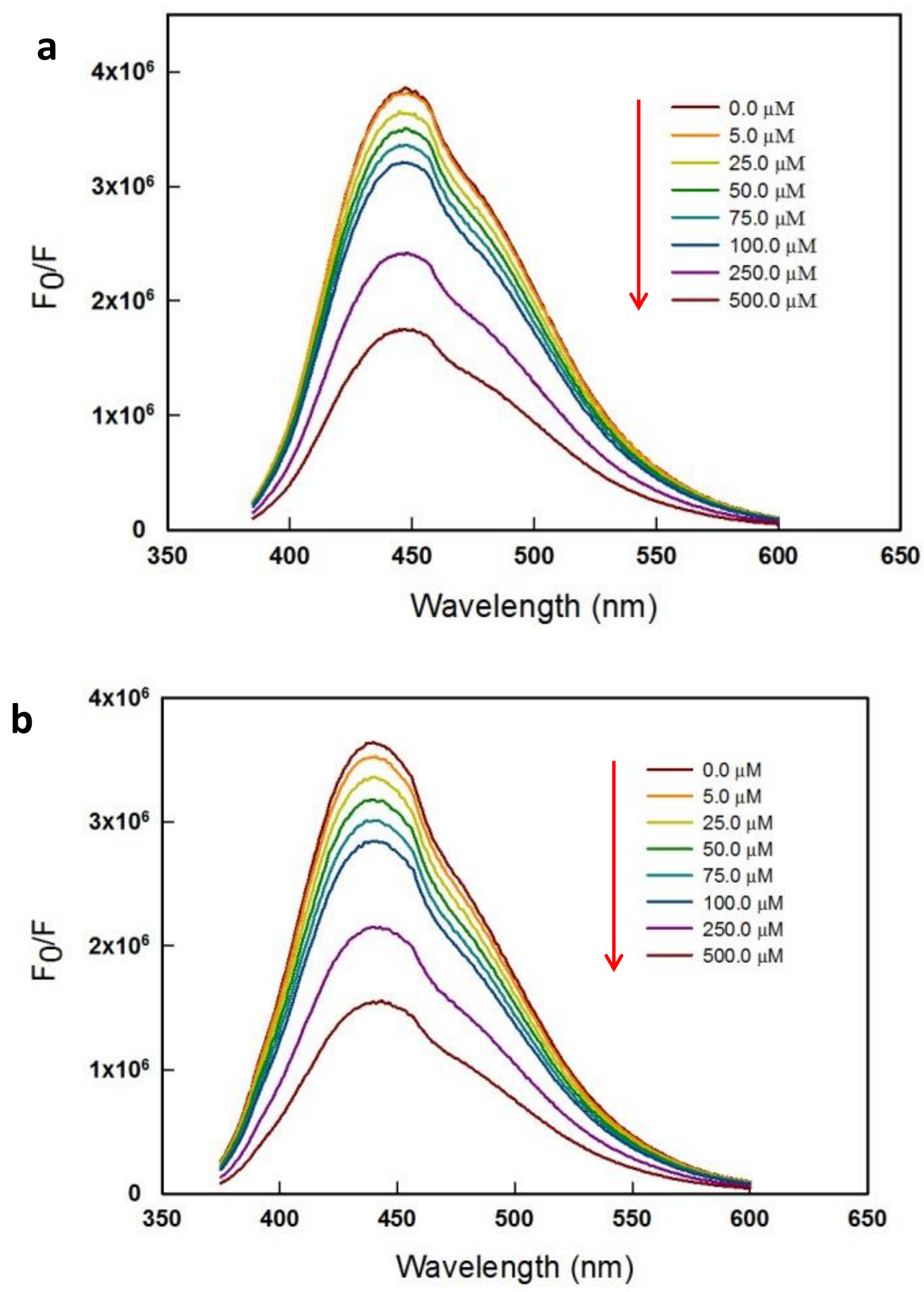

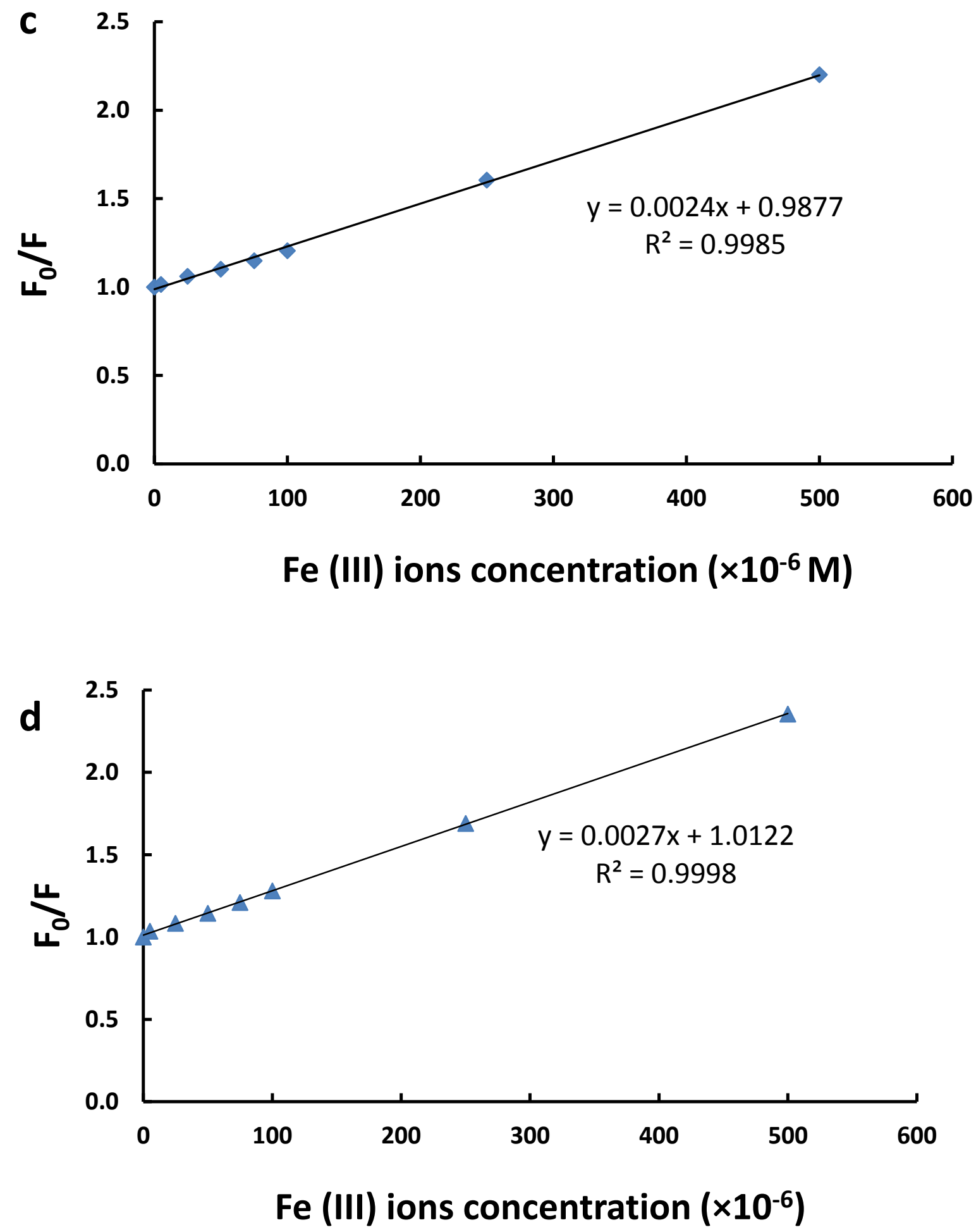

Figure 4.9 Fluorescence spectra of (a) T3000 C-dots and (b) T5000 C-dots responsing to the addition of $\mathrm{Fe}$ (III) ions in ultrapure water, and responding liner relationship 
between the concentrations of $\mathrm{Fe}$ (III) in ultrapure water and the relative fluorescence intensity of (c) T3000 C-dots and (d) T5000 C-dots, respectively

\begin{tabular}{|c|c|c|c|}
\hline C-dots & Peak $(\mathbf{n m})$ & $\mathbf{K s v}\left(\mathbf{M}^{-1}\right)$ & $\mathbf{R}^{\mathbf{1}}$ \\
\hline T3000 C-dots & 447 & $2.4 \times 10^{3}$ & 0.9985 \\
\hline T5000 C-dots & 440 & $2.7 \times 10^{3}$ & 0.9998 \\
\hline
\end{tabular}

Table 4.1 List of parameters for the Stern-Volmer fits to the T3000 and T5000 emission peaks in water.

\subsubsection{Ion Sensing in Sol-Gel Thin Films}

Sol-gel immobilization technique is one of the most promising optical sensor techniques because of their porous nature of the sol-gel network. The entrapped molecules are allowed to interact with external analytes and chemical species. ${ }^{[46]}$ Thin film derived by so-gel can be used to detect various molecules since they require short diffusion path and allow quick interaction and detection performance. The fluorescence properties of the JEFFAMINE ${ }^{\circ}$ T3000 and T5000 C-dots responding to this group of heavy metal ions was investigated by using the sol-gel thin film immobilization technique, where an large integrated gel network was polymerized from tetraethyl orthosilicate (TEOS), a widely used chemical precursors in sol-gel synthesis in the sol-gel process. ${ }^{[7]}$ Two main reactions are involved in the preparation of the sol-gel process. The first step is hydrolysis where TEOS firstly react with water and are catalyzed by acid which help increasing the hydrolysis rate. (shown in reaction (1)). In the second step condensation (reaction (2-3)), tons of siloxane bonds which are provided from the polymerization of water 
production are resulted from the hydrolysis step and form a porous network. (as illustrated by Figure 4.10). ${ }^{[8]}$

$$
\begin{gathered}
\mathrm{Si}-\mathrm{OR}+\mathrm{H}_{2} \mathrm{O} \stackrel{A C I D}{\longrightarrow} \mathrm{Si}-\mathrm{OH}+\mathrm{H}_{2} \mathrm{O} \\
\mathrm{Si}-\mathrm{OR}+\mathrm{Si}-\mathrm{HO} \rightarrow \mathrm{Si}-\mathrm{O}-\mathrm{Si}+\mathrm{ROH} \\
\mathrm{Si}-\mathrm{OH}+\mathrm{Si}-\mathrm{OH} \rightarrow \mathrm{Si}-\mathrm{O}-\mathrm{Si}+\mathrm{H}_{2} \mathrm{O}
\end{gathered}
$$
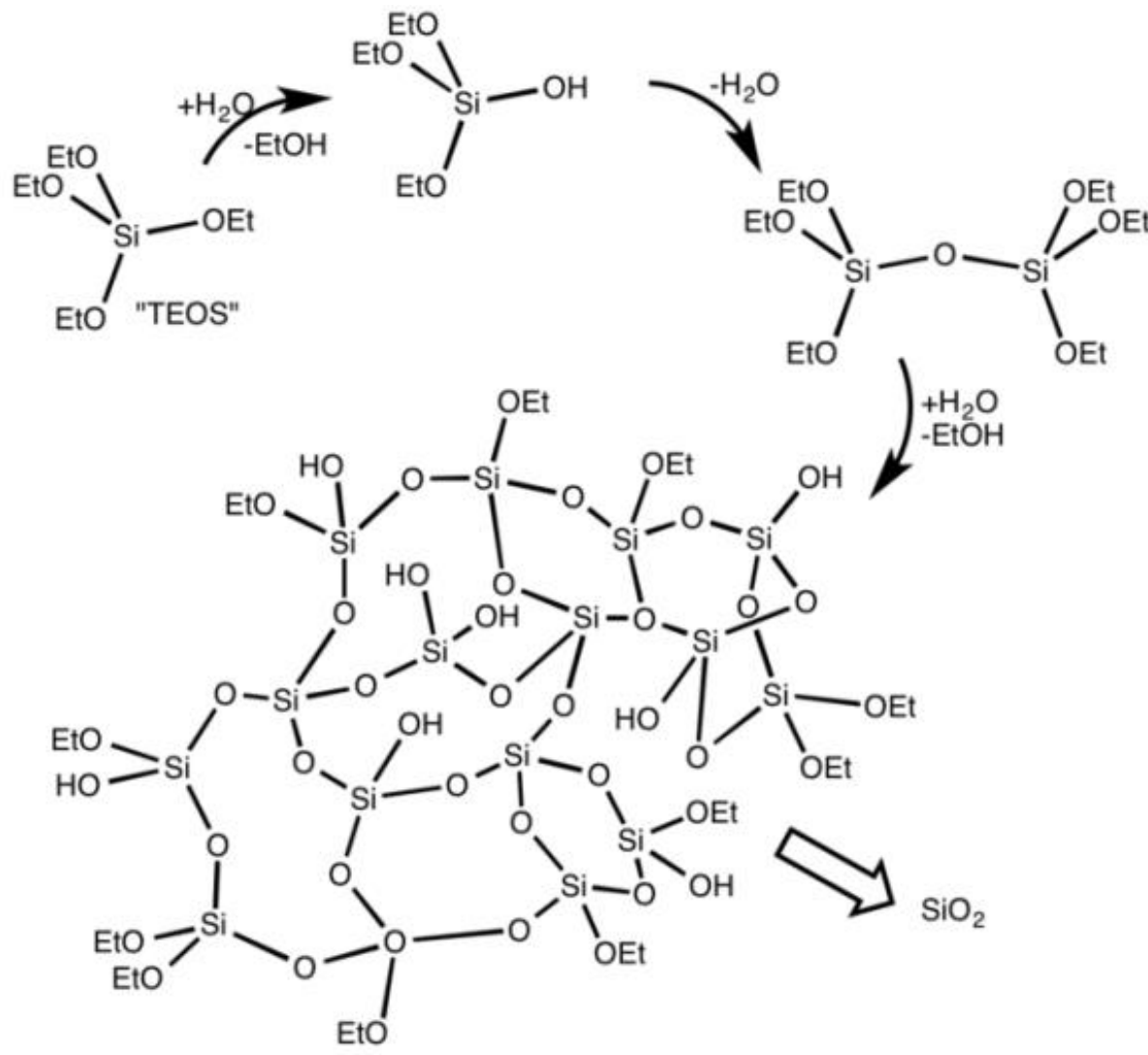

Figure 4.10 Scheme of condensation process of TEOS in sol-gel solution ${ }^{[11]}$

In order to explore the selectivity and sensitivity of the C-dots samples, FL intensity spectra from C-dots entrapped in sol-gel thin film were collected. The test was performed in ultrapure water. The sol-gel immobilized C-dots were prepared as followed: mixed C-dots and polymerized TEOS, coated on glass substrates and then immersed in ultrapure water. Possible interfere ions including $\mathrm{Sn}$ (II), $\mathrm{Hg}$ (II), 
$\mathrm{Cu}$ (II), Na (I), Ni (II), Zn (II), Mn (II), Sr (II) along with Fe (III) were tested by the sol-gel thin film system to investigated the selectivity. Increasing ion concentrations were added into the solution where sol-gel thin film was immersed with 15 minutes intervals and the fluorescence intensity was recorded as a result of the addition of the tested metal ion. However, we only analyzed points below $500 \mu \mathrm{M}$ in the sol-gel sensing research in order to get rid of the possible inner filter effect from the yellow colored Fe (III) ion solution. Figure 4.11 showed that the $500 \mu \mathrm{M} \mathrm{Ni}$ (II), $\mathrm{Na}$ (I), $\mathrm{Sr}$ (II) solution didn't quench the FL intensity of the entrapped T3000 C-dots, but increased fluorescence. Zn (II) had the strongest quenching effect, the relative intensity reached as high as 13.6. Fe (III) ions did on the emission of the T3000 C-dots in sol-gel thin film, Fe (III) ions typically showed consistent quenching sensitivity on entrapped T3000 C-dots with relative FL intensity $\left(F_{0} / F\right)$ of 2.1 , compared with the $F_{0} / F$ of $T 3000 \mathrm{C}$-dots quenched by 500 $\mu \mathrm{M} \mathrm{Fe}$ (III) ions in water which was 1.9. For the immobilized T5000 C-dots, Fe (III) showed much stronger quenching ability $\left(F_{0} / F=12.9\right)$ than it did upon T5000 Cdots in water $\left(\mathrm{F}_{0} / \mathrm{F}=1.8\right)$. However, $\mathrm{Na}$ (II), $\mathrm{Zn}$ (II), Mn (II) ions unexpectedly enhanced the fluorescence emitted by the T5000 C-dots in the thin film. 


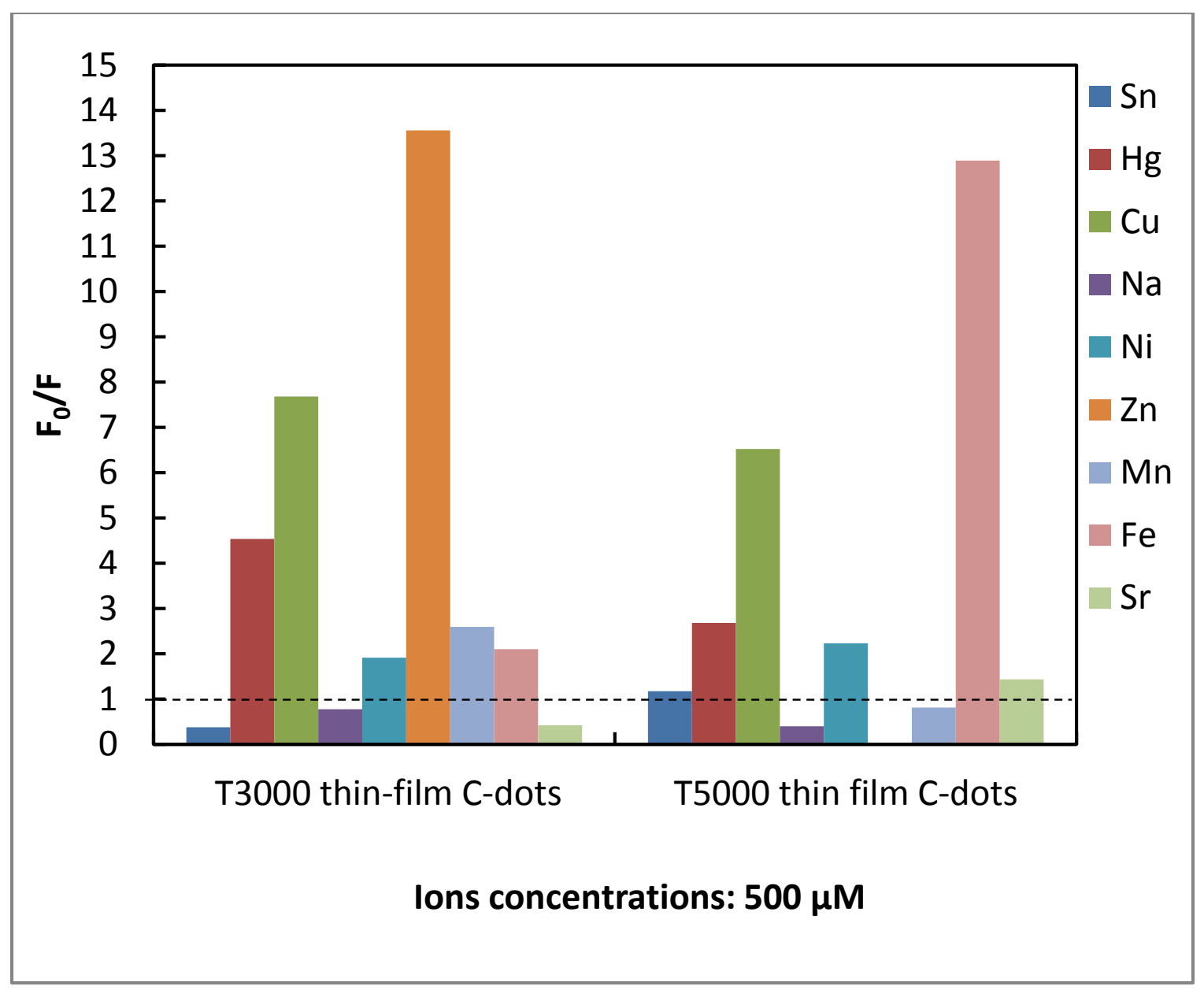

Figure 4.11 Quenching effect of various metal ions on relative fluorescence intensity $\left(F_{0} / F\right)$ of sol-gel entrapped T3000 and T5000 C-dots in ultrapure water

Apparently, the FL intensity response of the C-dots entrapped in sol-gel thin film towards the increasing metal ions concentrations was unstable. We entrapped Cdots within sol-gel thin films but doing so prevented the Fe (III) ions from quenching the T3000 C-dots. This is most likely due to the T3000 C-dots becoming inaccessible after thin film encapsulation. Furthermore, the random quenching effect of the 9 tested metal ions on the C-dots immobilized in sol-gel thin films might be explained by the following possibility: (1) Sol-gel matrix reduced the 
degree of freedom of the C-dots, and/or there was interactions between the $\mathrm{C}$-dots and the inner surface of the matrix pours. (2) The diffusion ability of metal ions was affected by the gel network. (3) The complexes formed by C-dots and Fe (III) ions were not stable and uniform. ${ }^{[47]}$

\subsection{Future Work}

Future work includes attempting the optimization of C-dots synthesis by microwave, such as shortening the heating-cooling cycles, trying other type of greener organic precursors etc, and the optimization of JEFFAMINE ${ }^{\circledR}$ T3000 and T5000 C-dots as applicable sensing materials immobilized in sol-gel thin film. For example, the solgel thin film will be aged for longer time before the fluorescence analysis; and some points below $500 \mu \mathrm{M}$ will be collected to evaluate the sensing ability of the entrapped C-dots. Moreover, effort should be devoted in understanding of the quenching mechanism performed in the solution and sol-gel thin films sensing system. For instance, UV-Vis absorption spectra and FTIR spectra will be collected after C-dots are in contact to the quencher in order to explore the change in the Cdots.

Another future work is to validating C-dots sensing system for the detection of $\mathrm{Fe}$ (III) ions in real sample to investigate their commercial potential. Furthermore, the reported so-gel thin film entrapped C-dots sensors can be coupled with optical fiber sensor to develop a small, reliable and economic analytical biosensor for the 
application of biological labeling, biomedicine, optoelectronic devices and sensors in the future.

\subsection{Conclusion}

In summary, we successfully synthesized high water-soluble carbon nanodots via the microwave-assisted method using JEFFAMINE ${ }^{\circledR}$ T3000 and T5000 as precursors. Without post surface passivation process, we have demonstrated that the one-step microwave-assisted method is a simple, rapid and green synthetic route for the production of water-soluble C-dots with highly intense photoluminescence $(\mathrm{PL})$. C-dots prepared through microwave pyrolysis were exhibited as uniform, spherical particles with small dimension and monodispersing in the solution in the TEM.

The prepared two types of C-dots displayed bright fluorescence under a UV-lamp and exhibited emission peaks centered at $448 \mathrm{~nm}$ and $439 \mathrm{~nm}$ respectively. Moreover, our study showed that JEFFAMINE ${ }^{\circledR}$ T3000 and T5000 C-dots have the feature of $\lambda_{e x}$ dependent emission. The resulting photoluminescence of excited Cdots was spectrally broad in visible spectrum, with peak intensity wavelengths ranging from $400 \mathrm{~nm}$ to $500 \mathrm{~nm}$ depending on the excitation wavelengths from 325 to $405 \mathrm{~nm}$.

The non-passivated JEFFAMINE ${ }^{\circledR}$ T3000 and T5000 C-dots synthesized by the one-step microwave-assisted route were also demonstrated to be useful as fluorescence sensor with their remarkably high selectivity and sensitivity of Fe (III) 
ions over other interfere metal ions. In this metal ions quenching study, the observed perfect linear relationship between the fluorescence intensity respond to the addition of Fe (III) ions (up to $500 \mu \mathrm{M}$ ) suggested that the JEFFAMINE ${ }^{\circledR} \mathrm{T}^{3000}$ and T5000 C-dots could potentially be applied as excellent sensor for the detection of Fe (III) ions with high sensitivity and selectivity. Meanwhile, we entrapped Cdots in sol-gel thin films and introduced a possible thin film sensing system for detecting metal ions in water, but will require further study in the future. Due to the advantages of low cost, easy preparation, highly sensitivity and sensitivity, T3000 and T5000 C-dots have the great potential to be developed as promising ecofriendly sensing probe in a real sample. 


\section{RFERENCE}

[1] C. Liu, P. Zhang, F. Tian, W. Li, F. Li, W. Liu, Journal of Materials Chemistry 2011, 21, 13163-13167.

[2] S. N. Baker, Baker, G. A., Angewandte Chemie 2010, 49, 19.

[3] aX. Michalet, F. F. Pinaud, L. A. Bentolila, J. M. Tsay, S. Doose, J. J. Li, G. Sundaresan, A. M. Wu, S. S. Gambhir, S. Weiss, Science 2005, 307, 538-544; bC. Y. Gao X, Levenson RM, Chung LW, Nie S, Nat Biotechnol 2004, 22, 8.

[4] aL. Cao, X. Wang, M. J. Meziani, F. Lu, H. Wang, P. G. Luo, Y. Lin, B. A. Harruff, L. M. Veca, D. Murray, S.-Y. Xie, Y.-P. Sun, Journal of the American Chemical Society 2007, 129, 11318-11319; bN. Na, T. Liu, S. Xu, Y. Zhang, D. He, L. Huang, J. Ouyang, Journal of Materials Chemistry B 2013, 1, 787.

[5] B. D. J. I. ZINK, Acc. Chem. Res. 2007, 40, 747-755, 40, 9.

[6] D. L. David Avnir, Renata Reisfeld J. Phys. Chem. 1984, 88, 4.

[7] D. A. P. Bulla, N. I. Morimoto, Thin Solid Films 1998, 334, 60-64.

[8] B. N. J. a. A. M. M. YOGESH S MHAISAGAR, Bulletin of Materials Science 2012, 35, 5 .

[9] S.K.Young, Army Research Lab Aberdeen Proving Ground 2002, MD 210055069,34

[10] aP. C. A. Jerónimo, A. N. Araújo, M. Conceição B.S.M. Montenegro, Talanta 2007, 72, 13-27; bD. B. Mitzi, Chem. Mater. 2001, 13, 16.

[11] Smokefoot, http://en.wikipedia.org/wiki/File:SolGelCartoon.png 2013.

[12] N. Innovations, http://www.nadetech.com/index.php/en/technologies.

[13] http://www.cpmt.org/mm/pkglab/theory/spin theory.html. 
[14] TyrionL, in http://commons.wikimedia.org/wiki/File:SolGel SpinCoating.jpg, Dec. 11. 2008 ed., 2008.

[15] A. S. S. C. Ray, Nikhil R. Jana,Rupa Sarkar, J. Phys. Chem. C 2009, 113, 5.

[16] Xiaoyou Xu, †, Robert Ray, †, Yunlong Gu, †, Harry J. Ploehn, †, Latha Gearheart, $\ddagger$, Kyle Raker, $\dagger$, and, W. A. Scrivens*, , J. AM. CHEM. SOC. 2004, 40, 2.

[17] B. Z. Ya-Ping Sun, Yi Lin, Wei Wang, K. A. Shiral Fernando, Pankaj Pathak,, B. A. H. Mohammed Jaouad Meziani, Xin Wang, Haifang Wang, Pengju G. Luo, $\dagger$, M. E. K. Hua Yang, Bailin Chen, L. Monica Veca, and Su-Yuan Xie, J. AM. CHEM. SOC. 2006, 128, 2.

[18] Q.-L. Zhao, Z.-L. Zhang, B.-H. Huang, J. Peng, M. Zhang, D.-W. Pang, Chemical communications 2008, 5116-5118.

[19] A. S. S. C. Ray, Nikhil R. Jana, Rupa Sarkar, 2009, 113, 5.

[20] D. G. Lei Tian, Wei Chen, Sulolit Pradhan, Xijun Chang, Shaowei Chen, Chem. Mater. 2009, 21, 6 .

[21] P.-C. Hsu, Z.-Y. Shih, C.-H. Lee, H.-T. Chang, Green Chemistry 2012, 14, 917.

[22] R. Hoogenboom, U. S. Schubert, Macromolecular Rapid Communications 2007, 28, 368-386.

[23] M. Tsuji, Hashimoto, M., Nishizawa, Y., Kubokawa, M., Tsuji, T., Chemistry 2005, $11,440-452$.

[24] J. T. Pelle Lidstrom, Bernard Wathey, Jacob Westman, Terahedron 2001, 59.

[25] T. F. S. MALLIKARJUNA N. NADAGOUdA, RAJENDER S. VARMA, Acc. Chem. Res. 2011, 44, 10.

[26] H. Zhu, X. Wang, Y. Li, Z. Wang, F. Yang, X. Yang, Chemical communications 2009, 0, 5118-5120.

[27] H. Peng, J. Travas-Sejdic, Chemistry of Materials 2009, 21, 5563-5565. 
[28] A. Safavi, Sedaghati, Fatemeh, Shahbaazi, Hamidreza, Farjami, Elaheh, RSC Advances 2012, 2, 7367.

[29] S. N. A. Mohd Yazid, S. F. Chin, S. C. Pang, S. M. Ng, Microchimica Acta 2012.

[30] L. Zhou, Y. Lin, Z. Huang, J. Ren, X. Qu, Chemical communications 2012, 48, 1147-1149.

[31] H. M. Goncalves, A. J. Duarte, J. C. Esteves da Silva, Biosensors \& bioelectronics 2010, 26, 1302-1306.

[32] A. Zhu, Q. Qu, X. Shao, B. Kong, Y. Tian, Angewandte Chemie 2012, 51, 71857189.

[33] Y. Mao, Y. Bao, D. Han, F. Li, L. Niu, Biosensors \& bioelectronics 2012, 38, 5560.

[34] O. L. I.Kuselman, Talanta 1993, 40, 8.

[35] N. V. R. a. T. W. O.S.Wolfbeis, Microchimica Acta 1992, 108, 9.

[36] T. L. Sally-Ann Wallington, Andrea Poppe, Nico A.J.M Sommerdijk, John D. Wright, Sensors and Actuators B: Chemical 1997, 38, 5.

[37] J. D. B. Kulwinder Flora, Anal. Chem. 1998, 70, 9.

[38] Y. Dong, R. Wang, G. Li, C. Chen, Y. Chi, G. Chen, Anal Chem 2012, 84, 62206224.

[39] V. F. ERNEST BEUTLER, TERRI GELBART,NGOC HO, DRUG METABOLISM AND DISPOSITION 2001, 29, 4.

[40] D. Wang, L. Wang, X. Dong, Z. Shi, J. Jin, Carbon 2012, 50, 2147-2154.

[41] B. Wang, J. Hai, Z. Liu, Q. Wang, Z. Yang, S. Sun, Angewandte Chemie International Edition 2010, 49, 4576-4579.

[42] J. Jiang, Y. He, S. Li, H. Cui, Chemical communications 2012, 48, 9634-9636.

[43] Y.-P. Sun, B. Zhou, Y. Lin, W. Wang, K. A. S. Fernando, P. Pathak, M. J. Meziani, B. A. Harruff, X. Wang, H. Wang, P. G. Luo, H. Yang, M. E. Kose, B. 
Chen, L. M. Veca, S.-Y. Xie, Journal of the American Chemical Society 2006, $128,7756-7757$.

[44] P. C. Hsu, H. T. Chang, Chemical communications 2012, 48, 3984-3986.

[45] B. Wang, J. Hai, Z. Liu, Q. Wang, Z. Yang, S. Sun, Angewandte Chemie 2010, 49, 4576-4579.

[46] K. K. Flora, J. D. Brennan, Chemistry of Materials 2001, 13, 4170-4179.

[47] aB. D. MacCraith, C. M. McDonagh, G. O'Keeffe, A. K. McEvoy, T. Butler, F. R. Sheridan, Sensors and Actuators B: Chemical 1995, 29, 51-57; bC. Malins, H. G. Glever, T. E. Keyes, J. G. Vos, W. J. Dressick, B. D. MacCraith, Sensors and Actuators B: Chemical 2000, 67, 89-95. 